\title{
User's Guide Virtual Hydropower Prospector Version 1.1
}

Douglas G. Hall Sera E. White Julie A. Brizzee Randy D. Lee

November 2005

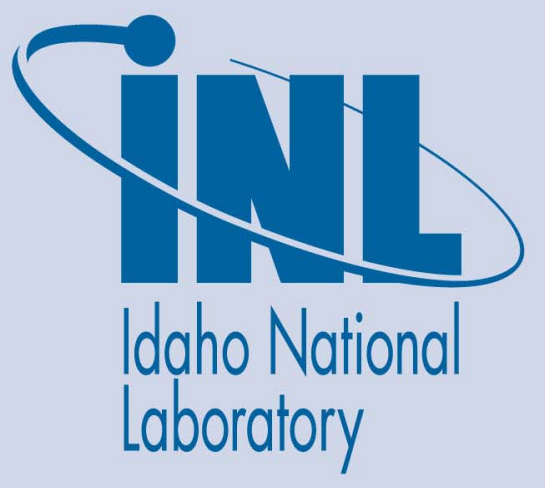

The INL is a U.S. Department of Energy National Laboratory operated by Battelle Energy Alliance 
INL/EXT-05-00402

Rev. 1

\section{User's Guide Virtual Hydropower Prospector Version 1.1}

Douglas G. Hall

Sera E. White

Julie A. Brizzee

Randy D. Lee

November 2005

Idaho National Laboratory
Idaho Falls, Idaho 83415

Prepared for the

U.S. Department of Energy

Assistant Secretary for Energy Efficiency and Renewable Energy

Under DOE Idaho Operations Office

Contract DE-AC07-05ID14517 


\section{ABSTRACT}

The Virtual Hydropower Prospector is a web-based geographic information system (GIS) application for displaying U.S. water energy resource sites and feasible, potential hydropower projects on hydrologic region maps. The application assists the user in locating sites of interest and performing preliminary, development feasibility assessments. These assessments are facilitated by displaying contextual features in addition to the water energy resource sites such as hydrography, roads, power infrastructure, populated places, and land use and control. This guide provides instructions for operating the application to select what features are displayed and the extent of the map view. It also provides tools for selecting features of particular interest and displaying their attribute information. 


\section{ACKNOWLEDGMENTS}

The authors acknowledge and express their appreciation of the contributions to the Virtual Hydropower Prospector made by the U. S. Geologic Survey National Center for Earth Resources Observation and Science by producing the Elevation Derivatives for National Applications dataset and the basic engineering data that was derived from it or used to produce data that is displayed by the application. The authors acknowledge and express their appreciation of data processing performed by Mr. Kelly Reeves, who standardized data and produced data displayed by the application. The authors acknowledge and express their appreciation of the various companies and agencies listed on the Data Sources webpage of the application for the application platform and data displayed by the application. 


\section{CONTENTS}

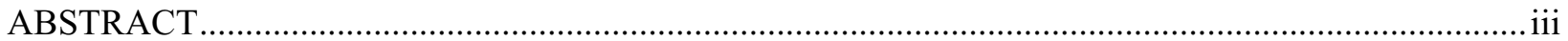

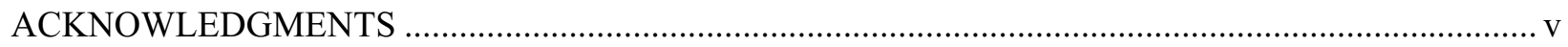

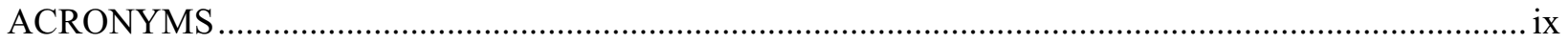

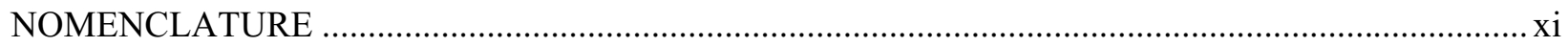

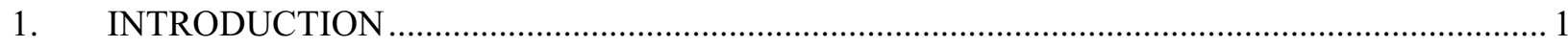

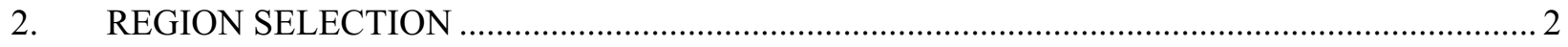

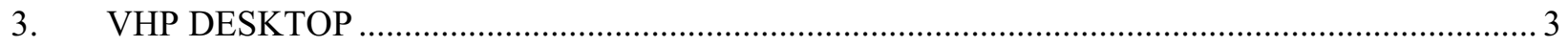

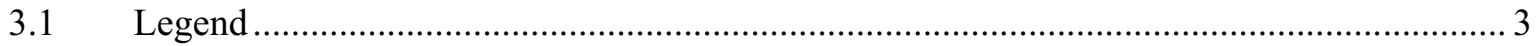

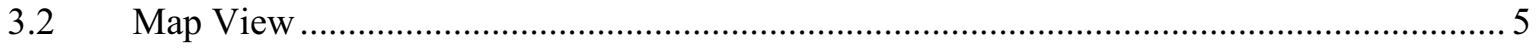

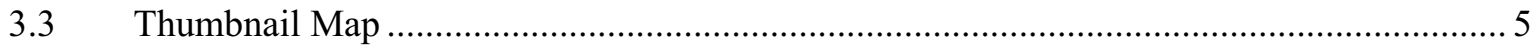

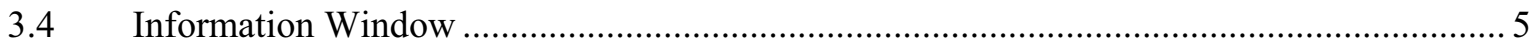

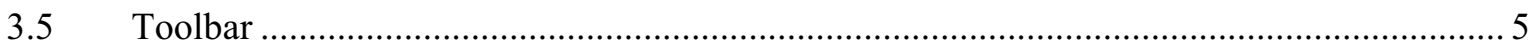

3.5.1 Access Buttons and Thumbnail Map Control ............................................... 6

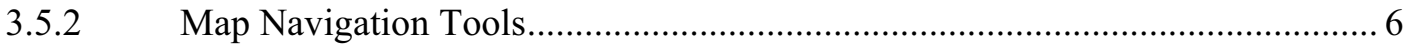

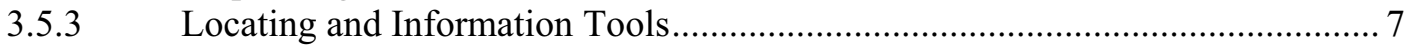

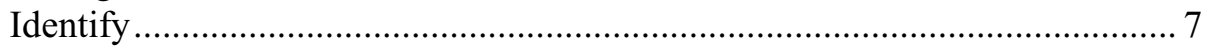

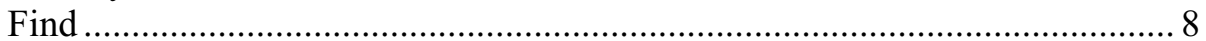

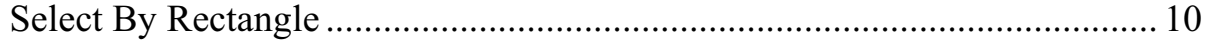

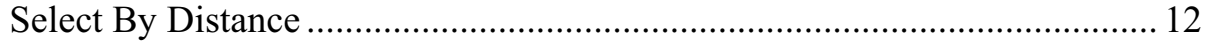

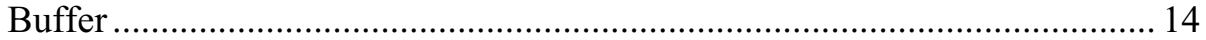

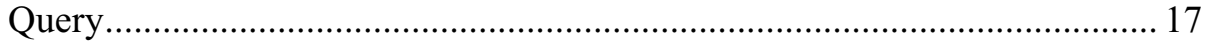

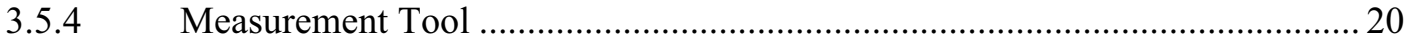

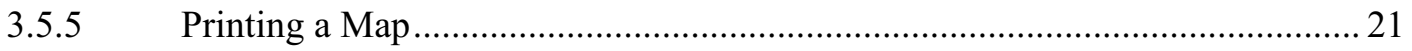

3.5.6 Including a Map Image in a Document or Slide .......................................... 23

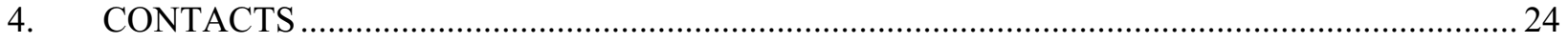

\section{FIGURES}

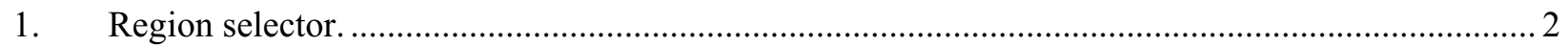

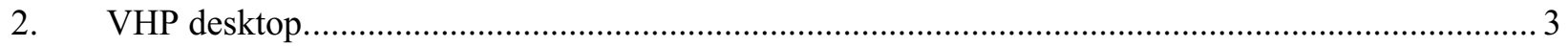

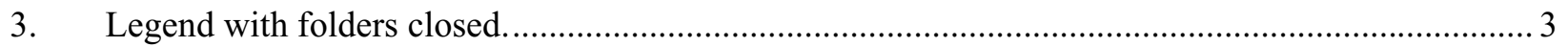

4. Part of the legend showing feature set selection using Feature Selection checkboxes..................... 4 


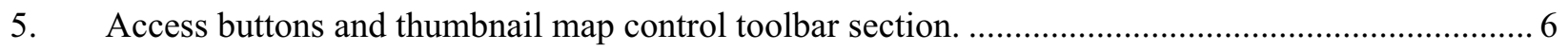

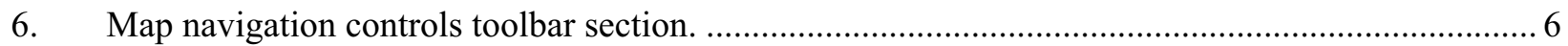

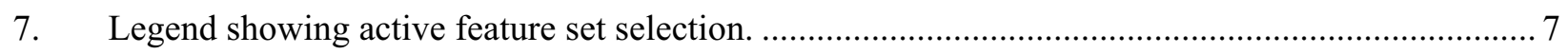

8. Example of information returned using the Identify tool. ...................................................... 8

9. Search information entry for the Find tool to locate the city of Ukiah, OR and display its

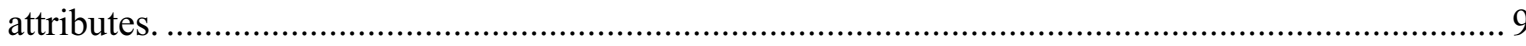

10. Information returned resulting from using the Find tool to locate the city of Ukiah, OR.............. 9

11. Map view after selecting Zoom to Feature and Highlight when locating the city of Ukiah,

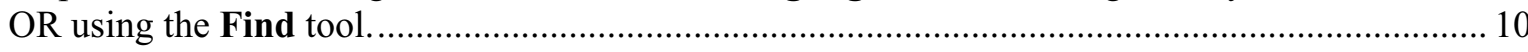

12. Map view after selecting a set of hydroelectric plants using the Select by Rectangle tool............ 11

13. Map view after selecting a particular hydroelectric plant from a set selected using the Select by Rectangle tool...

14. Selection circle radius entry for selecting all hydroelectric plants within 50 miles of the city of Denver, CO using the Select by Distance tool.

15. Map view resulting from selecting all hydroelectric plants within 50 miles of the city of Denver, CO using the Select by Distance tool.

16. Map view after using Highlight to locate the Sunshine hydroelectric plant from a group of plants within 50 miles of Denver, CO selected using the Select by Distance tool.

17. Form for entering select information for the Buffer tool.

18. Feature set list used to select feature set of target features for selection using the Buffer tool.

19. Map view of low power conventional turbine water energy resource sites within 2 miles of selected power lines selected using the Buffer tool.

20. Data form used to construct logic statements for selecting features using the Query tool.

21. Query data entry window illustrating complex query string to select high head/high power water energy resource sites having power potential $\geq 5 \mathrm{MW}$ and hydraulic heads $\leq 500 \mathrm{ft}$.

22. Map view and Information Window after selection of high head/high power water energy resource sites having power potential $\geq 5 \mathrm{MW}$ and hydraulic heads $\leq 500 \mathrm{ft}$ using the Query tool.

23. Measurement tool map view display and data readout window.................................................. 21

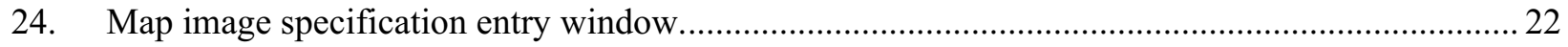

25. Sample map with title, legend, and thumbnail map produced by the Print tool. 


\section{ACRONYMS}

BIA Bureau of Indian Affairs

BLM Bureau of Land Management

BOR Bureau of Reclamation

DOD Department of Defense

DOE U.S. Department of Energy

EDNA Elevation Derivatives for National Applications

An analytically derived, three-dimensional dataset in which hydrologic features have been determined based on elevation data from the NED resulting in three-dimensional representations of "synthetic streams" (stream path coordinates plus corresponding elevations) and an associated catchment boundary for each synthetic reach (based on 1:24K-scale data for the conterminous United States and 1:63,360-scale data for Alaska) (Note: EDNA synthetic stream reaches do not uniformly coincide with NHD reaches. Conflation of EDNA and NHD features to improve the quality of both datasets is a later phase EDNA development.) (http://edna.usgs.gov)

EROS Earth Resources Observation Systems Data Center

ESRI Environmental Systems Research Institute

FERC Federal Energy Regulatory Commission

FS Forest Service

FWS Fish and Wildlife Service

GIS geographic information system

A set of digital geographic information, such as map layers and elevation data layers, that can be analyzed using both standardized data queries as well as spatial query techniques

HPRA Hydroelectric Power Resources Assessment

HUC hydrologic unit code

INL Idaho National Laboratory

NED National Elevation Dataset

A three-dimensional representation of topographic features composed of geographic coordinates on a 30-m grid with corresponding elevations that numerically represent the topography based on 1:24K-scale data for the conterminous United States and 1:63,360-scale data for Alaska (available for the entire United States from the U.S. Geological Survey). (http://ned.usgs.gov) 
NHD National Hydrography Dataset

A comprehensive set of digital spatial data that contain information about surface water features such as lakes, ponds, streams, rivers, springs, and wells. (http://nhd.usgs.gov)

NPS National Park Service

USGS U.S. Geological Survey 


\section{NOMENCLATURE}

Active Feature Set

Annual mean flow rate

Annual mean power

Attribute

Capacity

Catchment

Clicking

Drainage area

Drainage basin

Feasible potential project

Feature

Feature set
The feature set from which specific features are selected using the Information and Location tools in the VHP toolbar.

The statistical mean of the flow rates occurring at a particular location during the course of 1 year. The annual mean flow rates were estimated using regional flow regression equations based on gauged stream flow rates that occurred over a period of many years. The annual mean flow rate in any given year will usually differ from the value predicted by the equations.

A measure of the magnitude of a water energy resource's potential power producing capability equal to the statistical mean of the rate at which energy is produced over the course of 1 year. When based on the predicted annual mean flow rate and associated hydraulic head of a stream reach, the predicted annual mean power is the mean of the reach annual mean power that would occur over a period of many years. The actual annual mean power in a given year will usually differ from the predicted value of annual mean power.

A power rating of a hydroelectric plant based on electricity generation at this rate throughout the course of a year would produce the average annual electricity generation of the plant; sometimes referred to as average megawatt power rating denoted in some usages by "aMW."

Characteristic information about a feature such as name or owner, or data describing it such as length or voltage.

Typically refers to the design power rating of a hydroelectric plant and is on average equal to twice the annual mean power of the plant for existing United States hydroelectric plants.

The local drainage area surrounding a stream reach that provides runoff to the reach as opposed in flow entering the reach at its upstream end resulting from runoff from upstream catchments.

"Click" or "clicking" refers to depressing the left mouse button while the cursor is at a specified location. Also, a "right click" could be specified. In this case, the required action is to depress the right mouse button while the cursor is at a specified location.

The total surface area of the topography of a drainage basin.

The geographic area supplying runoff to a particular point on a stream equal to the area of all the catchments associated with upstream stream reaches supplying flow to the point.

A water energy resource site that has met a set of feasibility criteria project thus identifying it as feasible for development

A specific entity represented on a map by a point such as a city, a line such as a stream, or an area such as an Indian reservation.

A set of features of the same type such as cities or power plants. 
EDNA stream node

EDNA stream reach

Exclusion zone

Hydraulic head

Map server

Penstock

Pour point flow rate

Power

Power class
Starting point of an EDNA synthetic stream, a confluence on it or point of reference, or its terminus where it enters a saltwater body or a sink.

That portion of an EDNA synthetic stream between two EDNA stream nodes. (Note: Each stream reach has an associated local catchment and an associated drainage basin.)

An area in which development is highly unlikely due to federal land use statutes or policies or environmental constraints.

The elevation difference between the upstream and downstream ends of a column of water (such as in a penstock).

An internet-based application that displays geographic information on a map.

A pipe conducting water from the point of takeoff on a stream to a turbine.

The estimated flow rate of a stream reach equal to the runoff rate from the corresponding drainage basin.

The power potential of a feasible potential project based on its working flow rate and working hydraulic head.

The power and technology classes into which water energy resource sites have been divided based on their power potential:

- $\quad$ High Head/High Power

- Low Head/High Power

- High Head/Low Power

- Convention Turbine

- Unconventional Systems

- Microhydro

where high power refers to $\geq 1 \mathrm{MW}$, low power refers to $<1 \mathrm{MW}$, high head refers to $\geq 30 \mathrm{ft}$, and low head refers to $<30 \mathrm{ft}$. The conventional turbines, unconventional systems, and microhydro power technology classes are subclasses of the low power class defined by their operating envelopes as shown in the figure below.

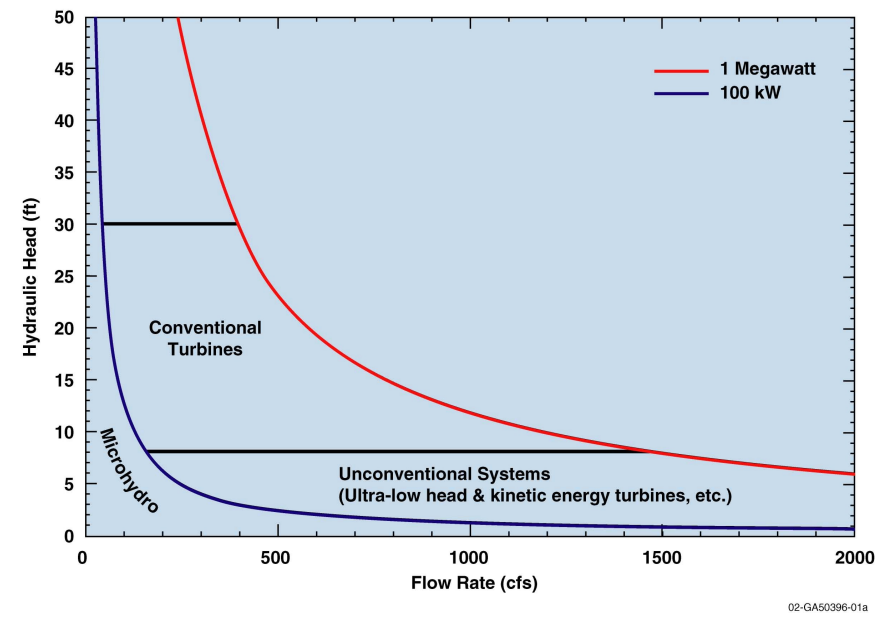


Power class

Power potential

Reach

Reach grade

Region

Right click

VHP desktop

Water energy

resource site

Working flow rate

Working head
The power and technology classes into which feasible potential projects have been divided based on their power potential:

- Small Hydro

- Low Head - Convention Turbines

- $\quad$ Low Head - Unconventional Systems

- Microhydro

where small hydro refers to $\leq 30 \mathrm{MW}$ and $\geq 1 \mathrm{MW}$, low power refers to $<1 \mathrm{MW}$. The conventional turbines, unconventional systems, and microhydro power technology classes are subclasses of the low power class defined by their operating envelopes as shown in the figure above except with no upper limit on hydraulic head for conventional turbines.

Ideal hydroelectric power based on an annual mean flow rate and an associated hydraulic head. The actual value in any given year will usually differ from the predicted value due to annual variations in annual mean flow rate. (Note: In the case of the developed power potential of an actual hydroelectric plant, the developed power potential is approximated by the annual mean power of the plant.)

A stream segment often delineated by two successive confluences.

The elevation difference between the upstream and downstream ends of the reach divided by the planimetric reach length (length of reach projection onto a horizon plane) expressed as a percentage $\left(100 \%\right.$ grade $=45^{\circ}$ slope $)$.

One of the 20 hydrologic regions into which the United States is divided, each composed of a set of drainage basins; in general, all flowing to the same stream or streams through which water flows out of the region. Regions are designated by hydrologic unit codes from 1 through 20 .

The action of depressing the right mouse button while the cursor is at a specified location.

The application desktop displayed in a single window and composed of the map view and controls for selecting the graphical and numerical information displayed by the application. (Note: Multiple windows each containing a complete VHP desktop devoted to a different hydrologic region may be open at the same time.)

A stream reach for which the values of hydraulic head, annual mean flow rate, and power potential have been estimated. The site location is taken as the longitudinal midpoint of the reach.

The rate of flow of water through a turbine.

The hydraulic head equal to the difference in the elevations of the entrance and exit of a penstock. 


\section{User's Guide Virtual Hydropower Prospector Version 1.1}

\section{INTRODUCTION}

The Virtual Hydropower Prospector (VHP) is a geographic information system (GIS) application designed to assist users in locating and assessing natural stream water energy resources in the United States. It was developed as part of the Small Hydropower Resource Assessment and Technology Development Project conducted at the Idaho National Laboratory (INL) in support of the U.S. Department of Energy Wind and Hydropower Technologies Program. The water energy resource sites displayed by this tool were identified and their gross potential power was estimated as described in Water Energy Resources of the United States with Emphasis on Low Head/Low Power Resources, DOE/ID11111, April 2004. The methods and criteria that were used to identify feasible potential hydropower projects and estimate their power potential using a set of development criteria are described in Feasibility Assessment of the Water Energy Resources of the United States for Low Power and Small Hydroelectric Plants, DOE/ID-11263, to be published January 2006.

The intended use of VHP is to obtain a broad overview of water energy resources in an area of interest or to perform a preliminary, development feasibility assessment of particular sites of interest. The location of features and the associated attribute information are for indication only. Actual on-site locations, measurements, and evaluations must be undertaken to verify information presented by VHP and assess true development feasibility

VHP was designed and developed using Environmental Systems Research Institute's (ESRI) commercial GIS software, ArcIMS 9.0, for the web and mapping interface. Additional functionality was developed in the Visual Studios InterDev programming environment. The application is served from an INL computer and does not require a user license to operate. The data displayed by the application are either publicly available or were purchased under a public use agreement.

This user's manual describes the five areas of the VHP desktop followed by explanations of the map view and map view or data access controls available in these areas, and the graphical or numerical information displayed in them. 


\section{REGION SELECTION}

The entry point into the VHP application is the Region Selector shown below in Figure 1. When you click near the region number of one of the 20 United States hydrologic regions, a new window opens that contains the VHP desktop with a map of the selected region in the map viewing area. The Region Selector can be accessed in three ways: 1) clicking on the Region Selector link located in the VHP homepage navigation alley, 2) clicking on the graphic on the VHP homepage, or 3) using the Region Selection button located in the toolbar on the VHP desktop.

The current version of VHP displays information only for a selected region. This ensures reasonable response times. However, if the area you wish to explore spans more than one region, you can chose to view multiple regions simultaneously. A new window with a VHP desktop is opened each time you click near a region number. Thus, you can view multiple regions by successively choosing between the windows you have opened, each displaying a different region. (Hint: Choosing the tile display option in the uppermost right corner of your computer screen may be a convenient way to cycle between VHP displays.)

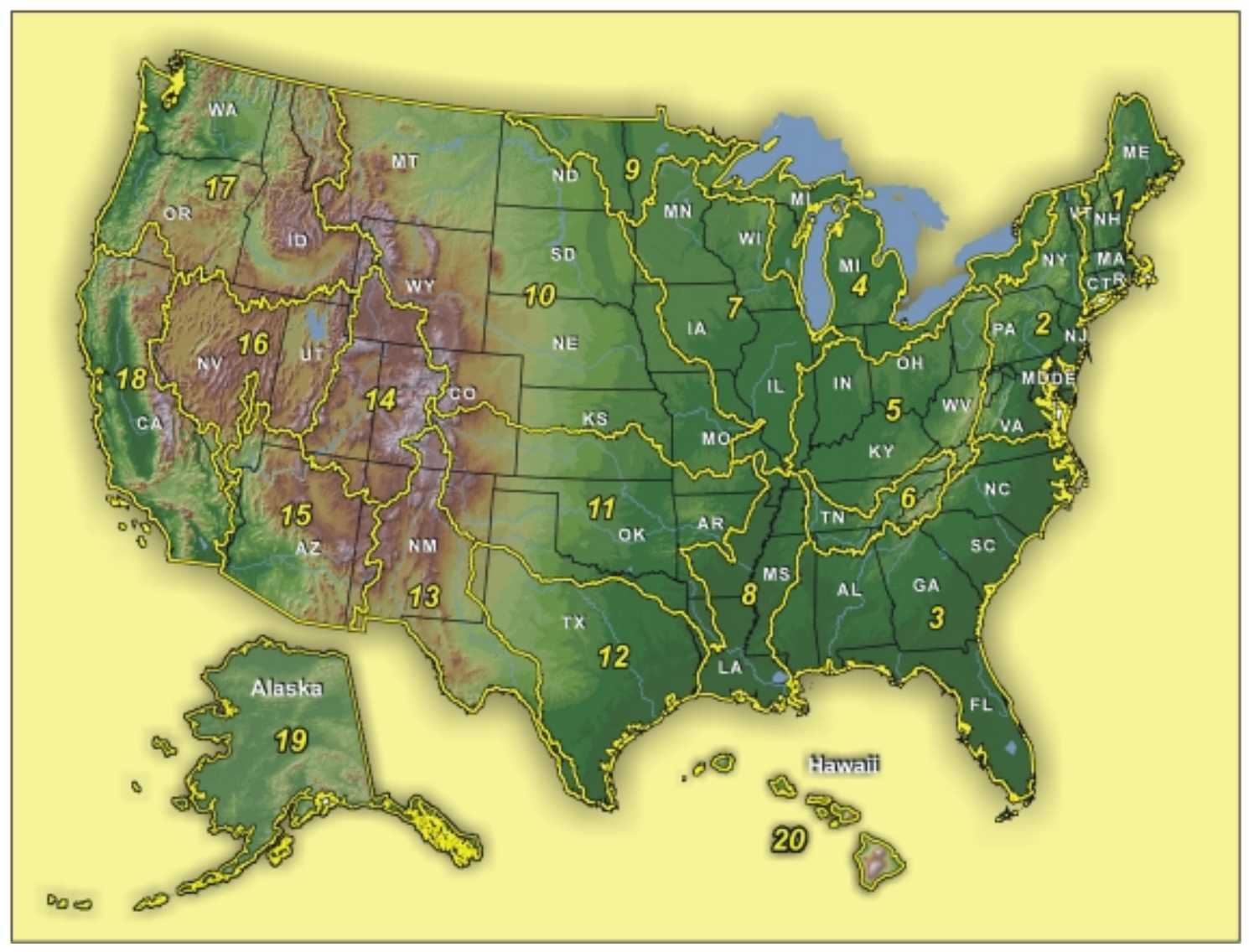

Figure 1. Region selector. 


\section{VHP DESKTOP}

Each VHP Desktop (Figure 2) consists of five areas:

- $\quad$ Legend

- $\quad$ Map View

- $\quad$ Thumbnail Map

- Information Window

- Toolbar

These areas and their associated functions are described in detail in the following sections.

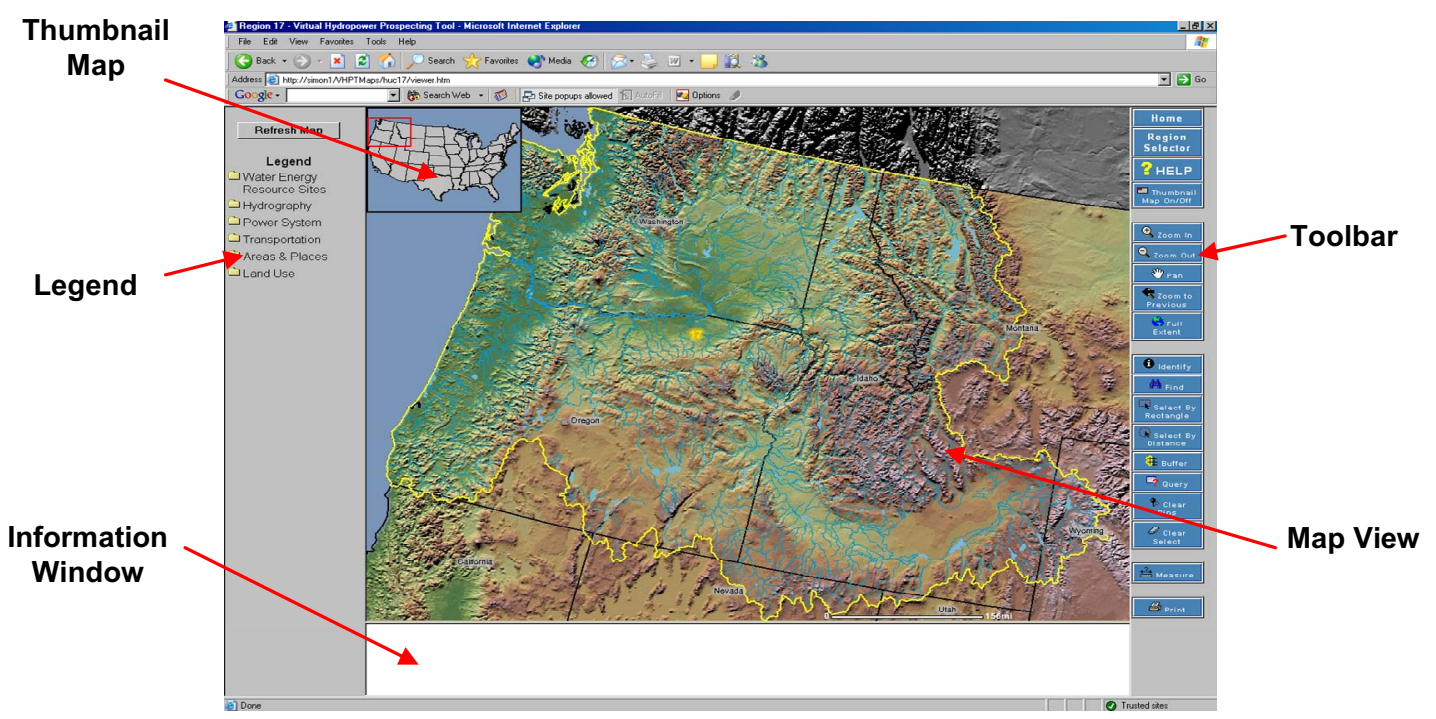

Figure 2. VHP desktop.

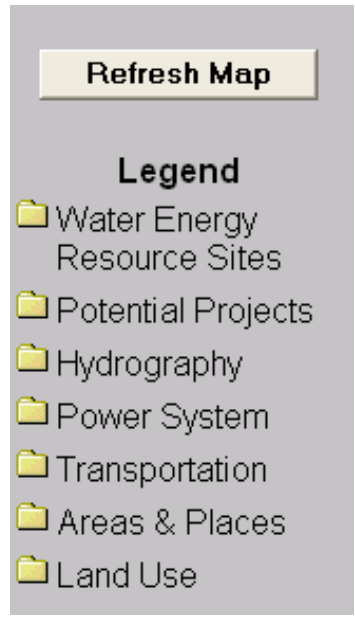

Figure 3. Legend with folders closed.

\subsection{Legend}

The Legend (Figure 3) is set up to function similar to a set of Windows folders. The folders each contain a group of feature sets that can be displayed on the map. Clicking on a closed folder opens it; revealing the feature sets it contains. Clicking on an open folder closes it; thus reducing the length of the legend.

The feature sets that can be displayed on the map are grouped by:

- Water Energy Resource Sites (High Head/High Power, Low Head/High Power, High Head/Low Power, Conventional Turbines, Unconventional Systems, Microhydro - see Nomenclature for definitions)

- Potential Projects (Small Hydro, Low Power Conventional Turbines, Low Power Unconventional Systems, Microhydro - see Nomenclature for definitions) 
- Hydrography (streams, waterbodies, Wild and Scenic Rivers, National Hydrography Dataset (NHD) streams, and Elevation Derivatives for National Applications (EDNA) streams.)

- $\quad$ Power System (Hydroelectric Plants, Other Power Plants, Power Lines, And Substations)

- $\quad$ Transportation (Primary Roads, All Roads, And Railroads)

- $\quad$ Areas \& Places (Cites, Populated Areas, County Boundaries, State Boundaries, Hydrologic Region Boundaries)

- $\quad$ Land use

- $\quad$ Excluded areas (areas where hydropower development is highly unlikely due to federal statutes and policies or environmental protections)

- $\quad$ Bureau of Indian Affairs (BIA)

- $\quad$ Bureau of Land Management (BLM)

- $\quad$ Bureau of Reclamation (BOR)

- $\quad$ Department of Defense (DOD)

- $\quad$ Forest Service (FS)

- $\quad$ Fish and Wildlife Service (FWS)

- $\quad$ National Park Service (NPS)

When initiated, the application displays only a few basic features on the map (hydrologic region boundary, state boundaries with state names, streams, and waterbodies). These features are indicated by a check mark in the feature selection box to the left of the feature set name (Figure 4). To display a feature set on the map, simply click its check box and then click the Refresh Map button. To remove a feature set from the map, click on its check box to remove the check mark and click the Refresh Map button.

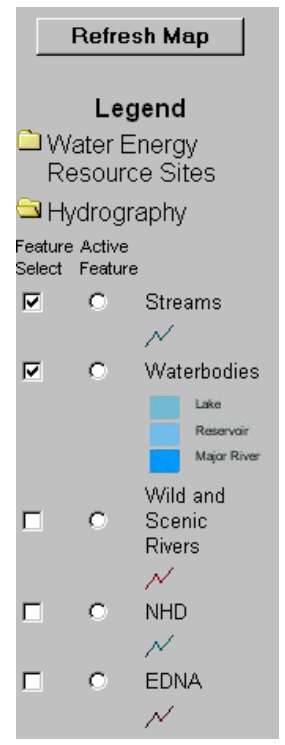

\section{IMPORTANT}

The Refresh Map button must be clicked each time the list of selected feature sets is changed by checking or unchecking one or more Feature Select boxes in the legend.

At present, opening or closing any legend folder clears all checks in Feature Select check boxes since the last map refresh, except those for the basic display feature sets. Therefore, you must click on the Refresh Map button after selecting feature sets for display in a folder before opening another folder, or open all the folders from which you want to select feature sets, select feature sets, and then click on the Refresh Map button.

Figure 4. Part of the legend showing feature set selection using Feature Selection checkboxes. 


\subsection{Map View}

Initially, the map view displays a map of the entire hydrologic region you selected using the Region Selector. The map at this point includes shaded relief with a nationwide, color-coded, elevation scale. It also includes only the hydrologic region boundary, state boundaries with state names, streams and waterbodies feature sets.

You will probably want to display other features on the map and "zoom in" to a smaller area on the map. What is displayed in the map view is controlled by the legend (see Section 3.1 above) and the map navigation controls (see Section 3.5.2 below). Additional feature sets are displayed by clicking on their Feature Select check box in the legend and clicking on the Refresh Map button.

\subsection{Thumbnail Map}

The thumbnail map located in the upper left corner of the map view shows what part of the conterminous U.S., Alaska, or Hawaii is being displayed in the map view. The red rectangle on the thumbnail map automatically changes size and location as you change the area being viewed using the map navigation controls like Zoom In, Zoom Out, and Pan. Display of the thumbnail map can be turned on and off by clicking on the Thumbnail Map On/Off button in the toolbar.

\subsection{Information Window}

The Information Window is primarily used to display a table of attribute information about features selected by one of the Locating and Information tools (see Section 3.5.3). Each row in the table provides attribute information about a specific feature. When using the Find or Select by Distance tools, clicking on a row in the table and then clicking on one of the locator buttons: Highlight, Insert Pin, or Zoom to Feature locates the feature on the map. When using the Select by Rectangle or Query tool, clicking on a row in the table automatically zooms the map image to the local area of the selected feature with its symbol located at the center of the map view and distinctly highlighted.

The Information Window also serves as a temporary data entry window for the Search by Distance, Buffer, and Query tools. This window is also used to enter specifications for map printing when using the Print tool.

\subsection{Toolbar}

The tool bar is located on the right side of the map view. It contains three sets of buttons, a button to activate the distance measuring tool, and a button to access the map image specification entry window. The three sets of buttons include:

- Access buttons and thumbnail map control

- Home

- $\quad$ Region Selector

- HELP

- Thumbnail Map On/Off

- Map navigation controls

- $\quad$ Zoom In

- $\quad$ Zoom Out 


\section{- Pan}

- Zoom to Previous

- $\quad$ Full Extent

- $\quad$ Locating and information tools

- Identify

- $\quad$ Find

- Select by Rectangle

- Select by Distance

- Buffer

- Query

- Clear Pins

- Clear Select

- $\quad$ Measure tool

- $\quad$ Print tool

\subsubsection{Access Buttons and Thumbnail Map Control}

\section{Home}

Region

Selector

? HELP

$\varpi$ Thumbnail

Map Onioff
The Home button provides access to the VHP homepage. The Region Selector button provides access to the Region Selector. The HELP button provides access to this User's Guide. The Thumbnail Map On/Off turns display of the thumbnail map on and off.

Figure 5. Access buttons and thumbnail map control toolbar section.

\subsubsection{Map Navigation Tools}

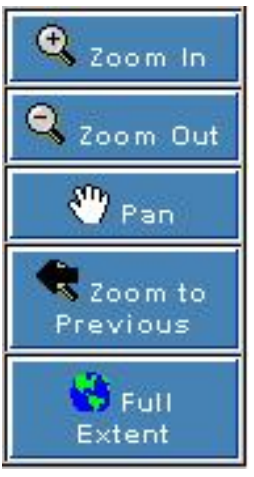

The map navigation controls are used to choose the area of the region map you want to view. Operating the controls as described below results in an automatic refresh of the map view with no change in the map feature set selections. However, some feature sets are zoom controlled and will not be displayed until a small enough area is viewed. Without zoom control, the symbols for very dense feature sets such as NHD hydrography and roads would completely obscure the symbols for all other features. In contrast to some features becoming visible when zooming in, topographic relief is not visible when zoomed in beyond a certain extent, because the background topography would become pixilated thus distorting the map view.

Figure 6. Map navigation controls toolbar section.

Clicking on Zoom In activates a cross $(+)$ cursor. There are two methods of zooming in to take a closer look at a smaller area of the map. When you click on the map, the view zooms in incrementally 
with each click. The resulting view is centered around the location you clicked on. The other method for zooming in is to click and hold in the map view to drag a rectangle around the area you want to view. When you release the mouse button, the map will automatically zoom into the area within the rectangle and centers it in the map view.

Zooming out to view a larger area of the region map than is currently displayed is accomplished by first clicking on the Zoom Out button. This activates a cross $(+)$ cursor. Placing the cursor on the map and clicking will zoom out the view with the resulting view centered on the cursor location when you clicked. Each time you click, the view zooms out and displays more area.

The area of the map in the map view can be "moved" like moving a map being viewed through a picture frame that only exposes part of the map. This capability is useful when the area you want to see is just "off the map" from the area you are currently viewing. Click the Pan button to "move" the map. This will activate a hand ( $(\mathbb{m})$ cursor. Click on the map and hold the mouse button down to drag the map in the desired direction to expose more of the map on the side opposite the direction you are dragging. This can be done successively to center the map view on the area you want to see. (Note that zoom does not change when you are panning.)

The remaining two buttons in the map navigation controls, Zoom to Previous and Full Extent, produce rapid reset to a previous map view. Clicking on the Zoom to Previous button returns the map view to the previous extent of zoom. Clicking on the Full Extent button returns the map view to the initial display of the entire hydrologic region.

\subsubsection{Locating and Information Tools}

The locating and information tools locate or provide information about specific selected features. This information is displayed in the Information Window at the bottom on the VHP map server. Specific

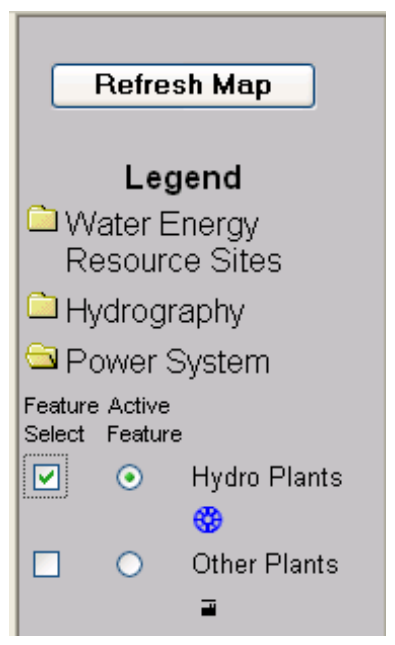
features can be located on the map by selecting an entry in the Information Window and zooming in on the area of the map where it is located, highlighting it, or by displaying a push pin symbol on it.

In order to use any of the locating and information tools, you must identify the feature set containing features you want to locate or about which you want detailed information. The feature set you choose is called the active feature set. The active feature set is selected in the Legend by clicking on the radio button next to the feature set name as illustrated in Figure 7. In this illustration, Hydro Plants has been selected as the active feature set. In addition to making the feature set the active set, you must also be sure that it is selected for display. If it is not, select it for display by checking its Feature Select checkbox and clicking the Refresh Map button.

Figure 7. Legend showing active feature set selection.

\section{(i) Identify}

The Identify tool is used to obtain detailed information about a specific feature in the active feature set. To use the Identify tool, do the following: 
1. First, be sure that the feature set containing features you want to identify or want information about is the active one and that it is selected for display. If it is not, check its Feature Selection checkbox and Active Feature radio button and refresh the map using the Refresh Map button.

2. Click on the Identify button to activate the tool.

3. Place the cursor on the specific feature you want information about and click on it.

The attribute information for the feature you selected will appear in the Information Window.

(Note: If there is a large area in the map view, it is possible to get information about more than one feature returned because the features are too close together to select just one precisely. In this case, you may want to zoom in more so that you can be sure to select just the one feature you want.)

An example of the information returned using the Identify tool and clicking on the Upper Little Sheep Creek Hydroelectric Plant is shown in Figure 8.

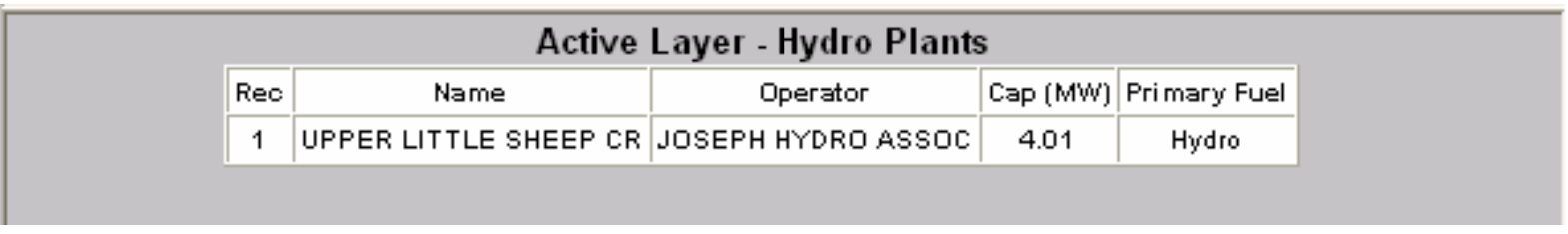

Figure 8. Example of information returned using the Identify tool.

The FIND tool will assist you in locating a feature on the map by performing a word search. Any feature in the active feature set having an attribute that contains the search string will be returned in the Information Window. To use the Find tool, do the following:

1. Be sure that the feature set you want to search is the active one and that it is selected for display. If it is not, check its Feature Selection checkbox and Active Feature radio button and refresh the map using the Refresh Map button.

2. Click on the Find button to activate the tool. A set of instructions will appear in the Information Window reminding you to select the active feature set and make it visible on the map and providing a place for you to enter the search string.

3. Enter the search string, which is not case sensitive.

4. Click on the Find button next to the search string window (not the Find button in the toolbar).

Figure 9 illustrates the use of the Find tool to locate the city of Ukiah, OR. 


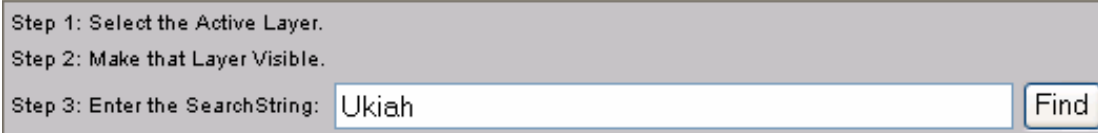

Figure 9. Search information entry for the Find tool to locate the city of Ukiah, OR and display its attributes.

Listings will appear in the Information Window for all of the features in the active feature set that have attribute information containing the search string. Figure 10 illustrates the attribute information displayed in the Information Window as a result of using the Find tool to locate the city of Ukiah, OR.

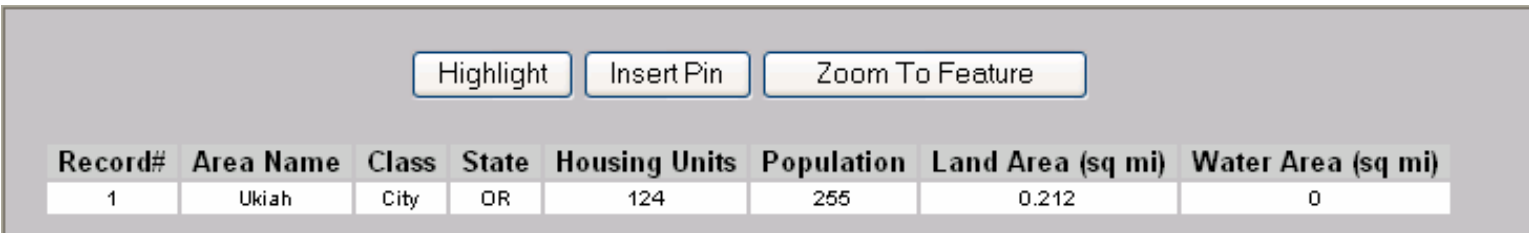

Figure 10. Information returned resulting from using the Find tool to locate the city of Ukiah, OR.

A particular feature listed in the Information Window can be located on the map in any of three ways: highlighting, displaying a pushpin symbol over it, or zooming to its location. To locate the feature on the map:

1. Click on its listing in the Information Window. The listing will be highlighted in blue.

2. Click on one of the locator buttons: Highlight, Insert Pin, or Zoom to Feature at the top of the Information Window.

Highlight will highlight the feature's symbol on the map with a red star. Insert Pin will display a pushpin symbol over the feature on the map. Zoom to Feature will zoom the map view to include the local area around the feature with the feature at the center of the view area. One or a combination of these locators can be activated by successively clicking on one of the locator buttons for the same highlighted listing in the Information Window. The result of selecting a combination of Zoom to Feature and Highlight in locating the city of Ukiah, OR is shown in Figure 11. 


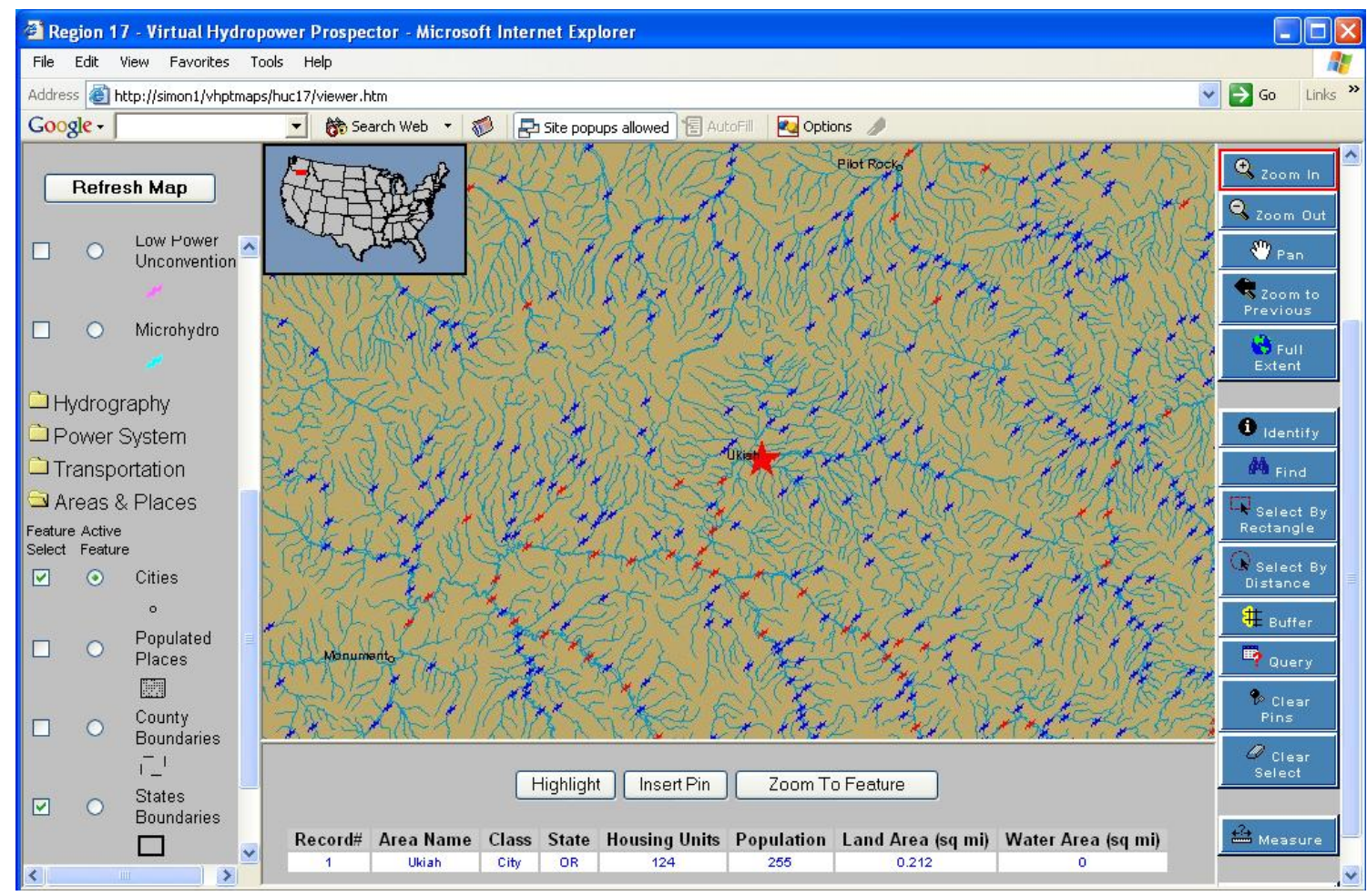

Figure 11. Map view after selecting Zoom to Feature and Highlight when locating the city of Ukiah, OR using the Find tool.

\section{The Clear Select button 1 seareat Pins button clears all of the pushpin images displayed on selected features.}

\section{Select By \\ Rectangle}

Select by Rectangle is used to select a set of features in the active feature set within a selected geographic area you define by drawing a rectangle on the map. To use the Select by Rectangle tool, do the following:

1. Be sure that the feature set containing the features you want to select is the active one and that it is selected for display. If it is not, check its Feature Selection checkbox and Active Feature radio button and refresh the map using the Refresh Map button.

2. Click on the Select by Rectangle button to activate the tool.

3. Place the cross $(+)$ cursor on the map at a position that will become the upper left corner of a rectangle.

4. Click and hold to drag a rectangle around the area containing the features you want to select. 
5. Release the mouse bottom and the map will automatically refresh with the features from the active feature set that are in the rectangle highlighted in larger, yellow, circle symbols. The attribute information for each of the features will appear in the Information Window.

The results of selecting a set of hydroelectric plants using the Select by Rectangle tool is shown in Figure 12.

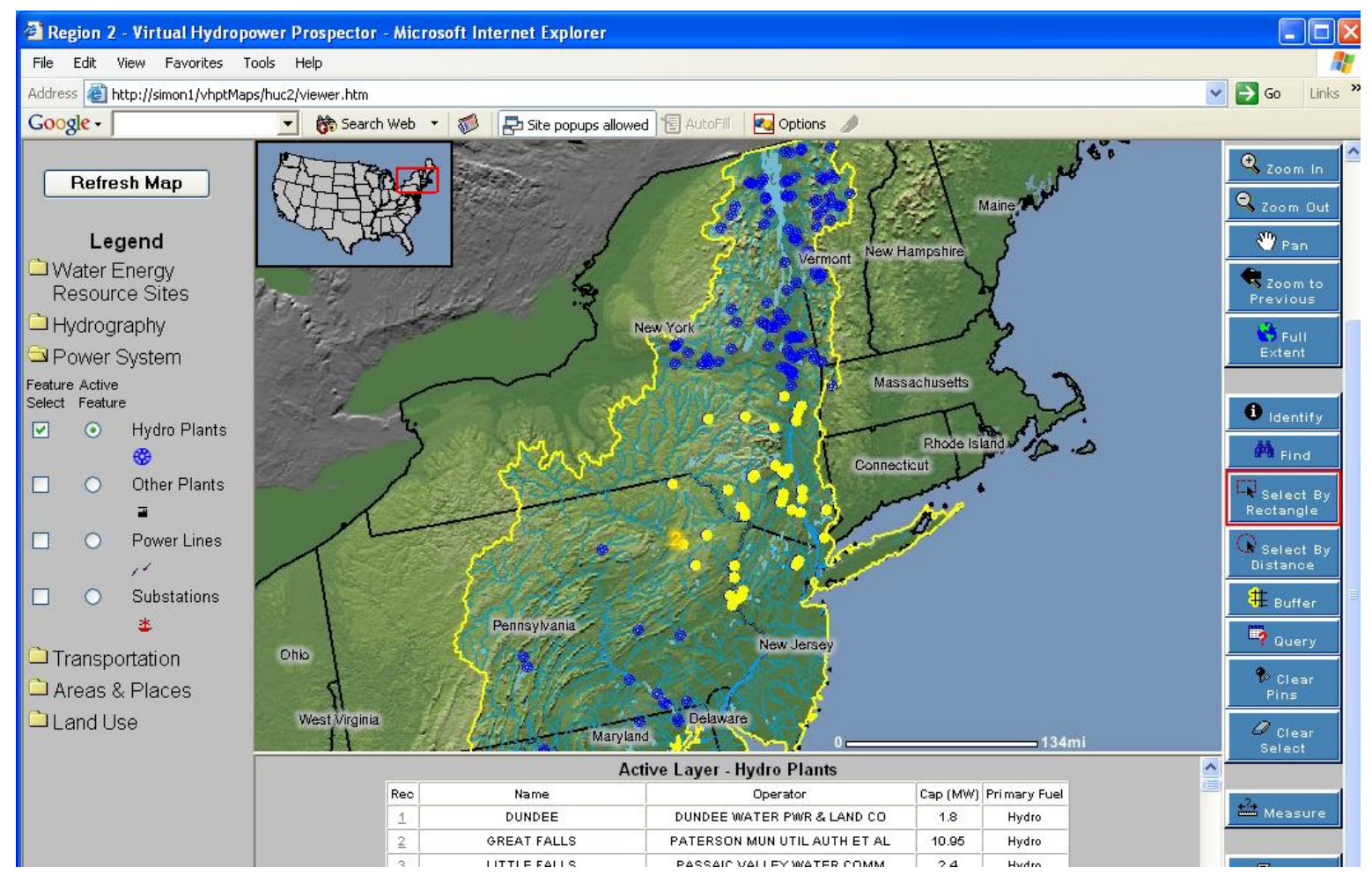

Figure 12. Map view after selecting a set of hydroelectric plants using the Select by Rectangle tool.

A particular feature listed in the Information Window can be located on the map by clicking on its listing in the Information Window. The map view will automatically zoom in on the area containing the selected feature, and it will be highlighted with a large red star at the center of the map view. The result of selecting a particular hydroelectric plant out of a set selected using Select by Rectangle is shown in Figure 13.

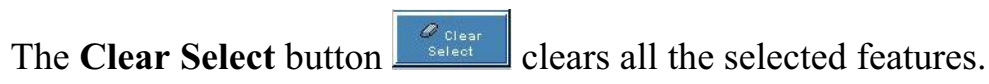




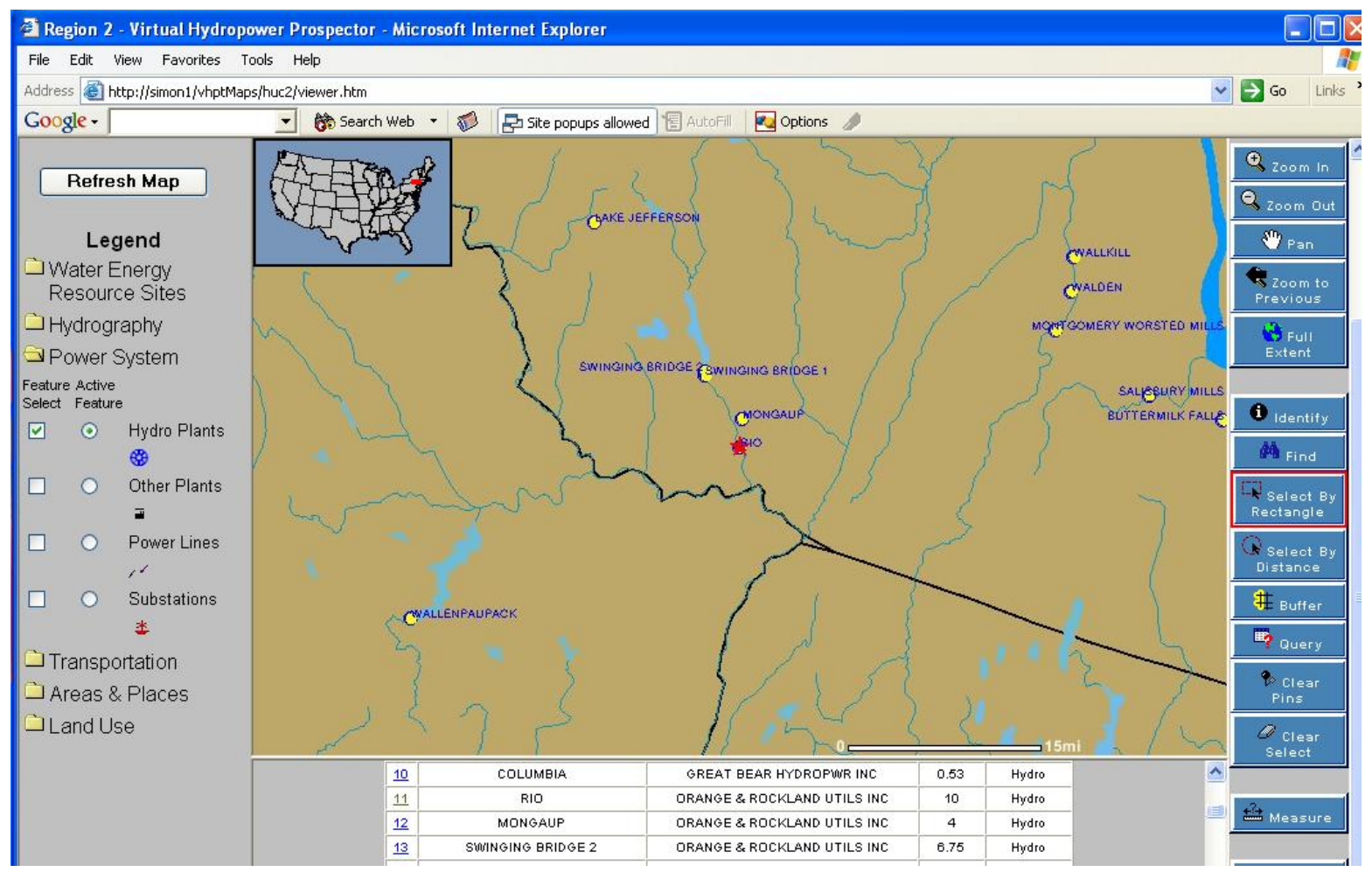

Figure 13. Map view after selecting a particular hydroelectric plant from a set selected using the Select by Rectangle tool.

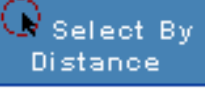

The Select by Distance tool is used to select features from the active feature set that are within a specified distance from a point on the map you select. To use the Select by Distance tool, do the following:

1. Be sure that the feature set containing the features you want to select is the active one and that it is selected for display. If it is not, check its Feature Selection checkbox and Active Feature radio button and refresh the map using the Refresh Map button.

2. Click on the Select by Distance button to activate the tool. The Information Window will display a data window labeled "Radius Distance: $\square$ miles."

3. Click on the data window and enter the number of miles you want the search radius to be.

4. Click on the checkbox labeled "Draw the selection circle," if you want the selection circle to be displayed on the map.

5. Place the cross $(+)$ cursor on the map at a position that will become the center of the selection circle and click.

Entry of the search radius to select the hydroelectric plants within 50 miles of the city of Denver, CO is shown in Figure 14. 


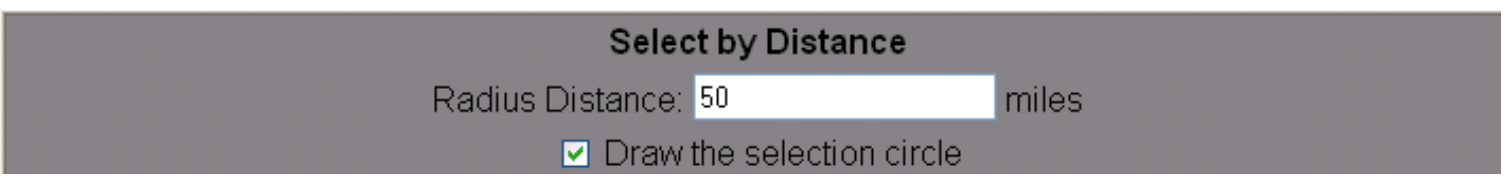

Figure 14. Selection circle radius entry for selecting all hydroelectric plants within 50 miles of the city of Denver, CO using the Select by Distance tool.

When you click on a point on the map, two things happen:

1. All of the features in the active features set that are within the distance you specified from your selected point will be highlighted. If you checked the Draw the selection circle checkbox, a shaded circle centered on the point on the map you clicked on will be present on the map showing the selection circle.

2. The information attributes for all the highlighted features will appear in the Information Window.

The map view following selection of all hydroelectric plants within 50 miles of the city of Denver, CO is shown in Figure 15.

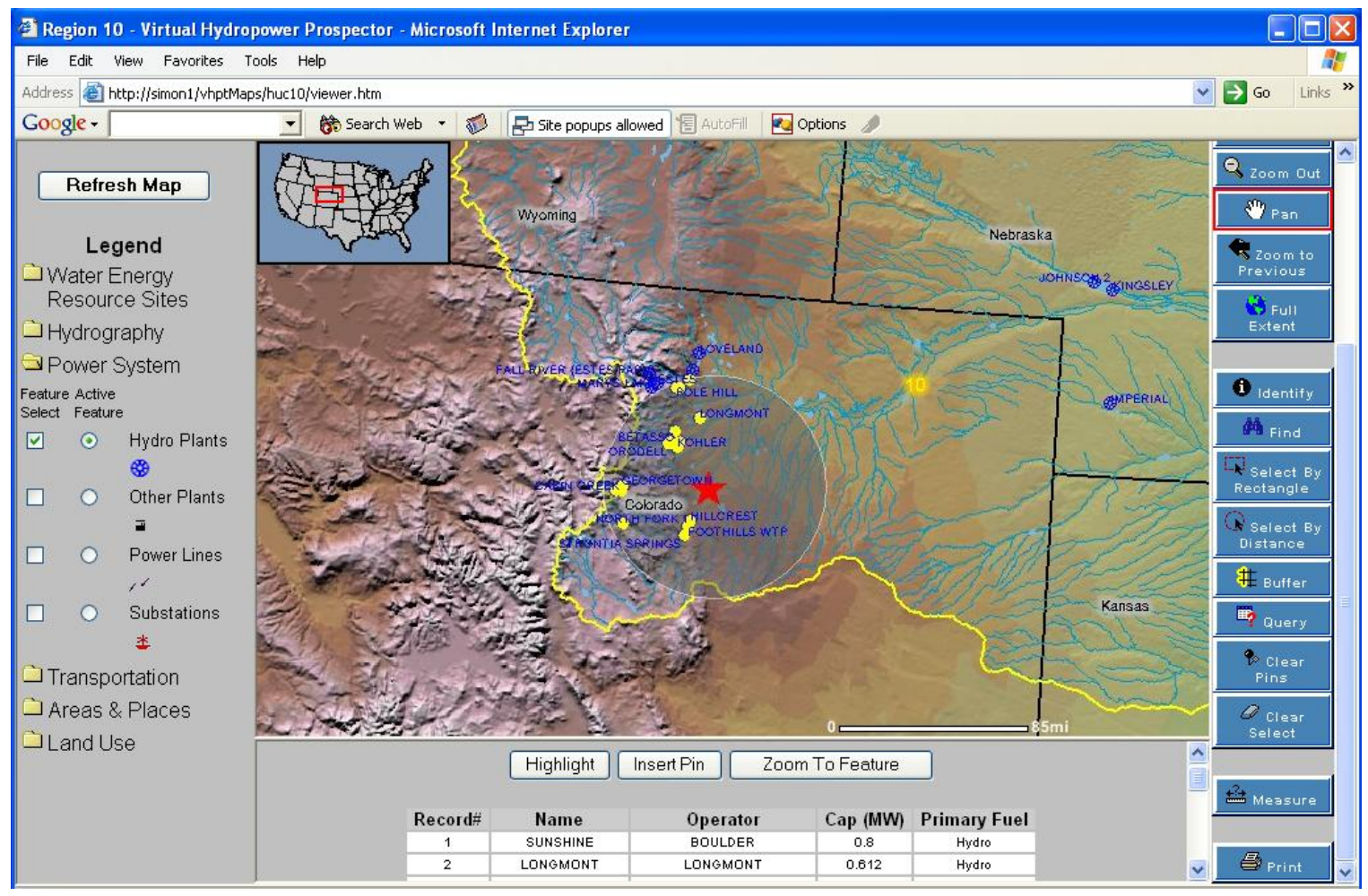

Figure 15. Map view resulting from selecting all hydroelectric plants within 50 miles of the city of Denver, CO using the Select by Distance tool.

A particular feature listed in the Information Window can be located on the map in any of three ways: highlighting, displaying a pushpin symbol over it, or zooming to its location. To locate the feature on the map:

1. Click on its listing in the Information Window. The listing will be highlighted in blue. 
2. Click on one of the locator buttons: Highlight, Insert Pin, or Zoom to Feature at the top of the Information Window.

Highlight will highlight the feature's symbol on the map with a red star. Insert Pin will display a pushpin symbol over the feature on the map. Zoom to Feature will zoom the map view to include the local area around the feature with the feature at the center of the view area. One or a combination of these locators can be activated by successively clicking on one of the locator buttons for the same highlighted listing in the Information Window. The result of locating the Sunshine hydroelectric plant using the Highlight locator is shown in Figure 16.

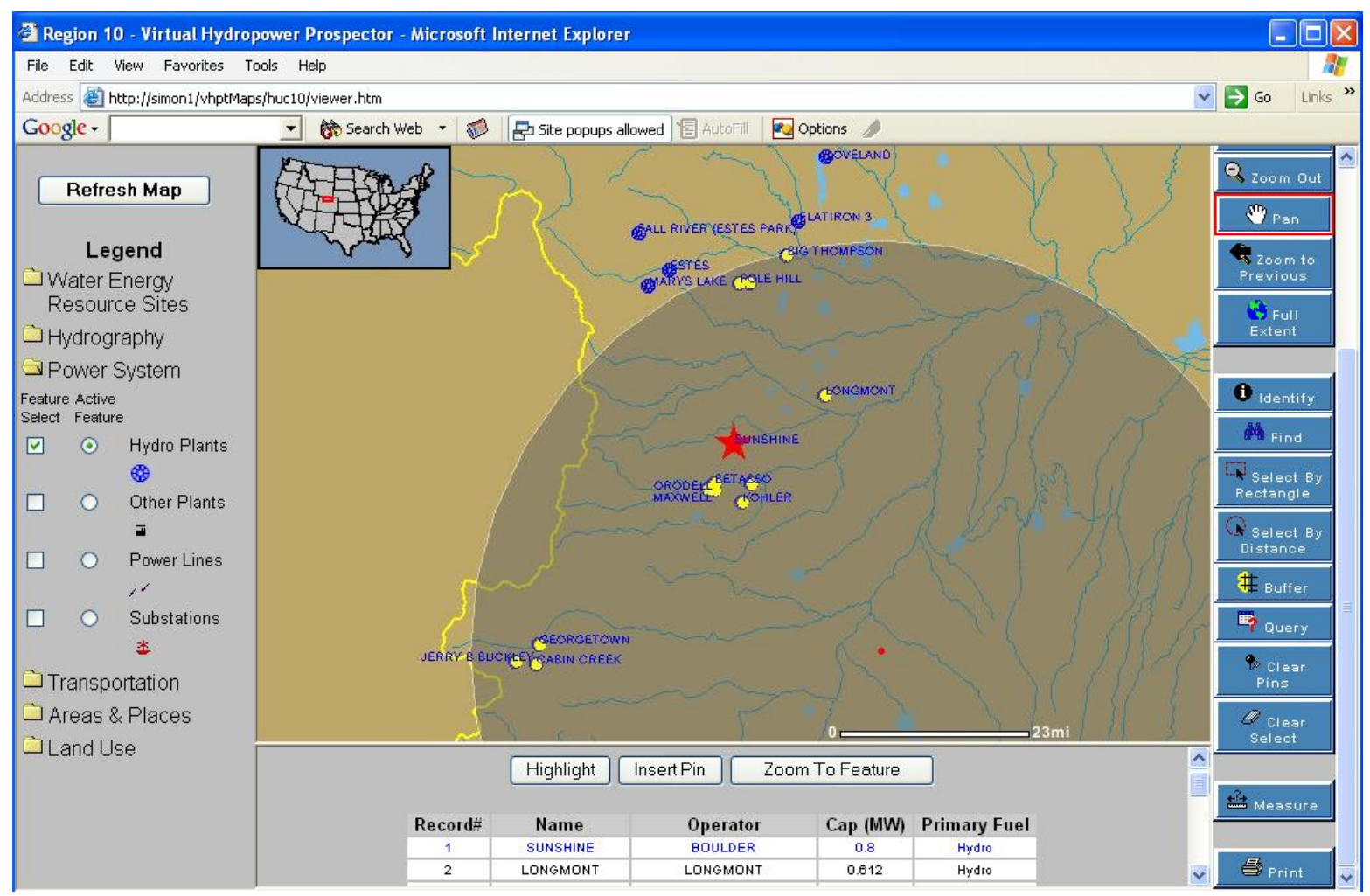

Figure 16. Map view after using Highlight to locate the Sunshine hydroelectric plant from a group of plants within 50 miles of Denver, CO selected using the Select by Distance tool.

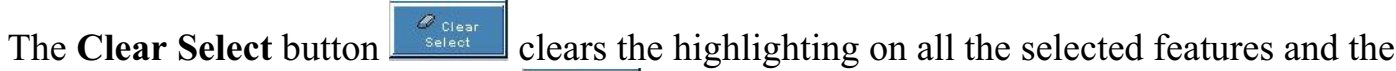

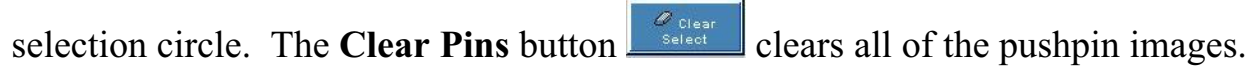

\section{\# Buffer}

The Buffer selection tool is an expanded version of the Select by Distance tool. With this tool, you can select features within a specified search distance (buffer) of another feature or group of features. The reference feature or features from which you are looking for other features can be a point on the map like a power plant, a line on the map like a transmission line, or an area feature on the map like a metropolitan area (populated place). To use the Buffer tool, do the following:

1. Select a feature or group of features using the Find, Select by Rectangle, Select by Distance. or Query tools. 
2. Click on the Buffer button to activate the tool. The Information Window will display a data window like the one shown in Figure 17.

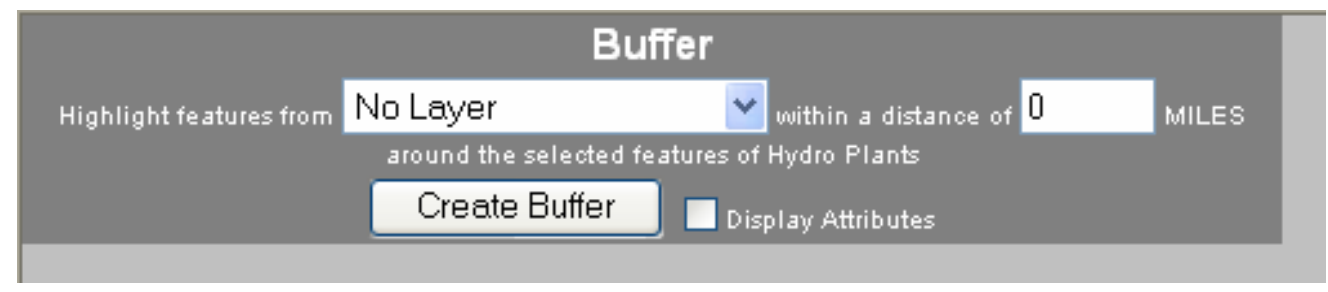

Figure 17. Form for entering select information for the Buffer tool.

3. The first data window in the form is preceded by "Highlight features from".

a. Click on the checkmark at the right of the data window to open a list of feature sets.

(Figure 18 shows the result of opening the list of feature sets. These are all the feature sets in the Legend.)

b. Click on the feature set containing the features you want to select using the Buffer tool.

4. The second data window in the form is preceded by "within a distance of". Enter a distance in miles in this data window. The tool will search for features of the type you selected in Step 3 in a search area (buffer) this distance from every part of the reference feature or features you selected in Step 1.

Note that the remainder of the statement containing the two data windows referred to in Steps 3 and 4 reads, "around the selected features of $X X X$ " where $X X X$ is the feature set containing the feature or features you selected in Step 1.

5. Click on the Display Attributes box if you want the information attributes of selected features displayed in the Information Window.

6. Click on the Create Buffer button. 


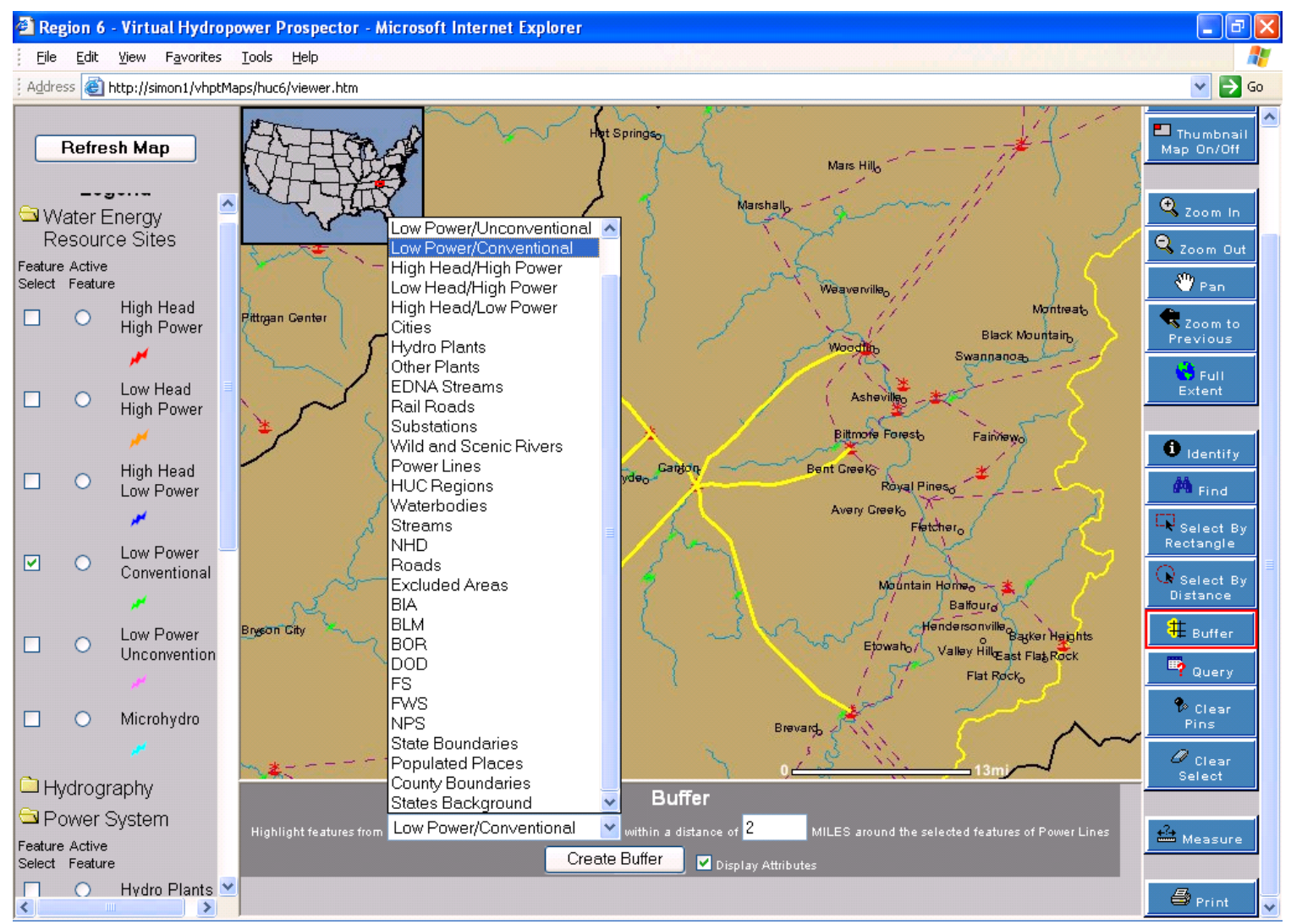

Figure 18. Feature set list used to select feature set of target features for selection using the Buffer tool.

When you click the Create Buffer button several things happen:

1. A buffer zone having the distance you selected will appear around the reference feature or features in the map.

2. The features in the feature set you selected in Step 3 that are within the number of miles you entered in Step 4 of the reference feature or features you selected in Step 1 will be highlighted in red on the map. (Note: In order to view all of the selected features it may be necessary to use Zoom Out until the entire extent of the buffer zone or zones is visible in the map view.)

3. If you checked the "Display Attributes" checkbox, attribute information listings for each of the selected features will appear in the Information Window.

An example of selecting low power convention turbine water energy resource sites within 2 miles of a set of power lines using the Buffer tool is shown in Figure 19. zones.

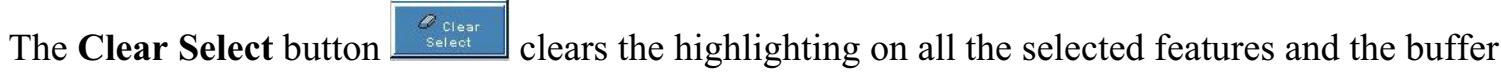




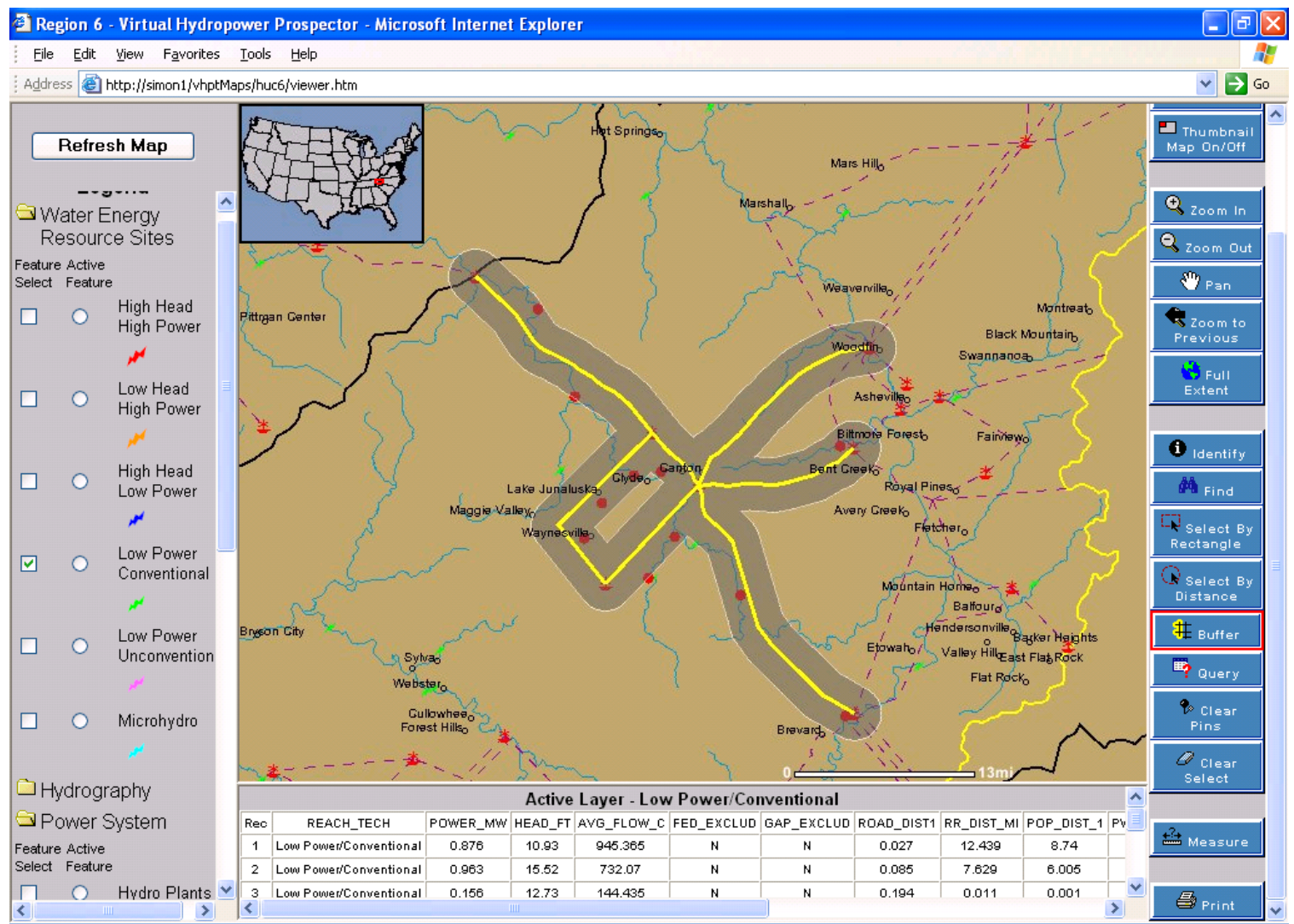

Figure 19. Map view of low power conventional turbine water energy resource sites within 2 miles of selected power lines selected using the Buffer tool.

\section{哭 Query}

The Query tool is used to select features using a logic statement written using the attributes of the features in the active feature set. A complex logic statement can be constructed using the tool. To use the tool, do the following:

1. Be sure that the feature set containing the features you want to select is the active one and that it is selected for display. If it is not, check its Feature Selection checkbox and Active Feature radio button and refresh the map using the Refresh Map button.

2. Click on the Query button to activate the tool. The Information Window will display the form shown in Figure 20. 


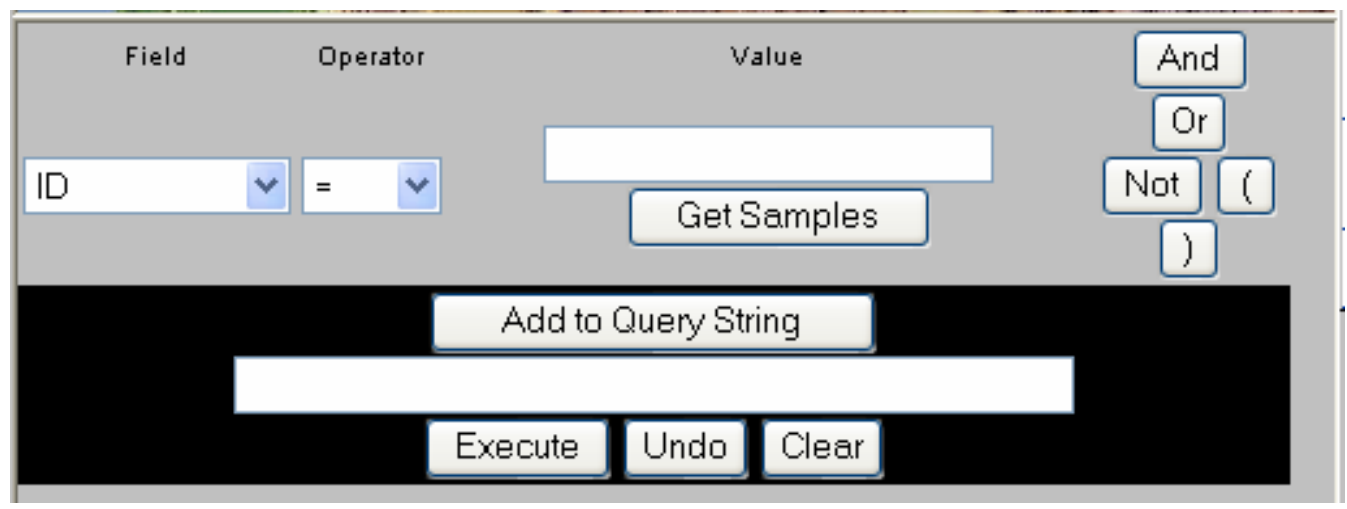

Figure 20. Data form used to construct logic statements for selecting features using the Query tool.

3. Click on the check mark next to the "Field" text box. This opens a window containing all attribute names associated with the active feature set. Choose one of these names by clicking on it. It will be the subject of the query statement.

4. Unless equals $(=)$ is the operator you want, click on the check mark next to the "Operator" text box. This will open a window containing all of the operators. Chose one of the operators by clicking on it.

5. To complete the query statement, enter a value in the "Value" data window.

Note: If you are not sure what a realistic valve is to enter in the data window, click on Get Samples button. This will open a window containing representative values of the attribute you have selected as the subject of your query. Click on a value close to the one you want. At this point, you cannot edit the data value, but you will be able to after the query statement is added to the query string in the next step.

Note: If you enter a numeric value or edit a representative value, the number entered must have the same number of digits to the right of the decimal place as the numbers in the representative values. Without this conformance, the query will fail and return an error message.

6. When you are ready to accept the three parts of the query statement, click on the Add to Query String button. This will move the query statement you constructed to the query string window below the Add to Query String button.

7. Edit the query string, if necessary.

Note: You can change the operator you chose by editing it in the query string window. However, the operator must be edited to look like one of the operators shown in the Operator window. Editing the Field name is not recommended because the names used in query statements are not always identical to those shown in the Field data window. To change the Field name, click the Undo or Clear buttons at the bottom of the query data entry window and re-enter the query statement.

8. If you only need a simple query string, click the Execute button to activate feature selection according to the query string. 
When you execute the selection by query string, two things happen:

1. All of the features in the active features set for which the query string you constructed is true will be highlighted in the map view.

2. The information attributes for all the highlighted features will appear in the Information Window.

A particular feature listed in the Information Window can be located on the map by clicking on its listing in the Information Window. The map view will automatically zoom in on the area containing the selected feature, and it will be highlighted with a large, red, circle symbol at the center of the map view.

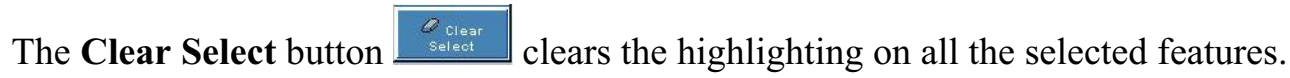

If you want to enter a complex query string, do the following:

1. Enter the first query string as described above.

2. Chose a conjunction by clicking on one of the conjunction buttons: And, Or, Not. The conjunction will be added to the query string.

3. Construct another query statement as described above.

4. Add the query statement to the query string by clicking on the Add to Query String button.

Note: The actions of the Add to Query String button and conjunction buttons (And, Or, Not and parentheses) can be undone using the Undo button. The Clear button will clear the entire query string.

5. When you have constructed the complete query string and it reads the way you want it to, click on the Execute button to activate feature selection according to the query statement.

The query data entry window for selecting all of the high head/high power water energy resource sites in Region 16 having power potentials greater than or equal to $5 \mathrm{MW}$ and hydraulic heads less than or equal to $500 \mathrm{ft}$. is shown in Figure 21.

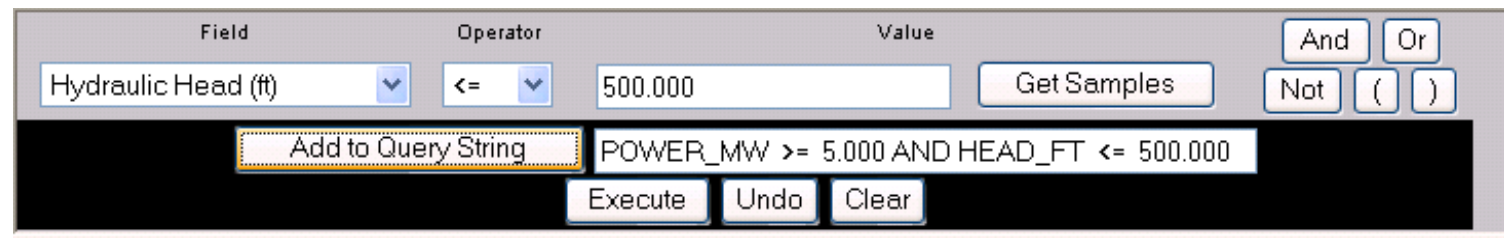

Figure 21. Query data entry window illustrating complex query string to select high head/high power water energy resource sites having power potential $\geq 5 \mathrm{MW}$ and hydraulic heads $\leq 500 \mathrm{ft}$.

The results of this query selection are shown in Figure 22. High head/high power water energy resource sites for which the query string is true are highlighted in yellow in the map view and the Information Window lists the attributes of each of the highlighted sites.

You can zoom in on the area in which the selected records are located by clicking on "Zoom to these records" at the bottom of attribute table in the Information Window. Depending on the location of the selected features, the map view will zoom in or out. 


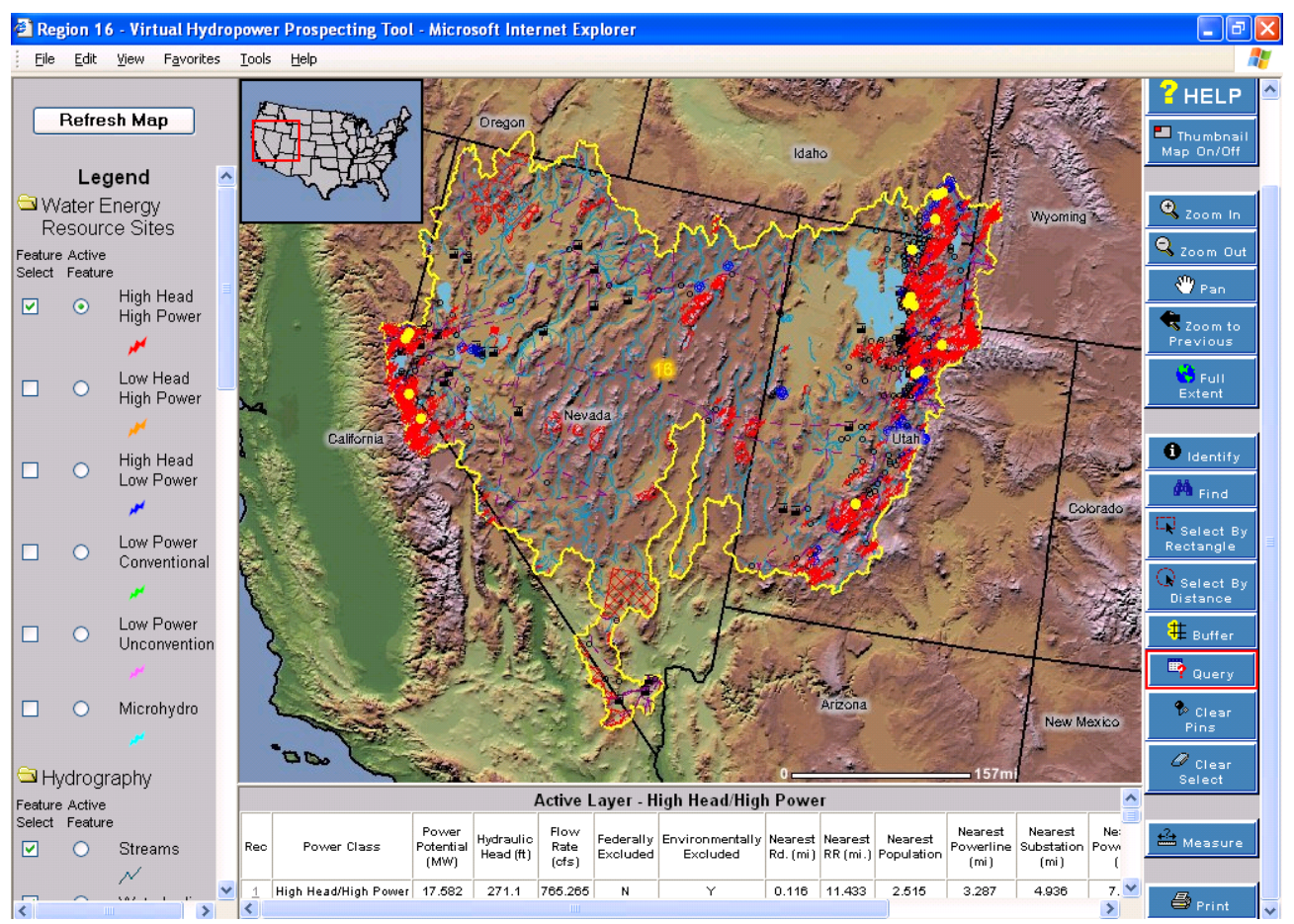

Figure 22. Map view and Information Window after selection of high head/high power water energy resource sites having power potential $\geq 5 \mathrm{MW}$ and hydraulic heads $\leq 500 \mathrm{ft}$ using the Query tool.

\subsubsection{Measurement Tool}

\section{㥂 Measure}

The Measure tool is used to measure the distance between two points on the map. It can also be used to measure the cumulative distance of a set of successive points you select on the map. To use the tool, do the following:

1. Click on the Measure button to activate the tool. A measurement data window will appear at the top of the map view.

2. Click on the point of the map you want to measure from. After you have selected this reference point, the distance between this point and wherever the cursor is moved on the map is readout in "Segment" data window in the measurement data window. This allows you to compare distances from a reference point or feature to other surrounding points or features.

3. To record the distance between the reference point and another point on the map, click on the point you want to measure to. The tool will draw a line on the map between these two points and the distance between them will appear in the "Total" data window in the measurement data window.

4. If you would like to measure the distance between the second point you clicked on in Step 3 and a third point, move the cursor to this third point and click. (Note: Because you clicked on the second point, it is now the reference point and the distance between this point and wherever the cursor is on the map is readout in the "Segment" window.) When you click on the third point, the tool draws 
a line segment between the second and third points and the total distance between the three points is displayed in the "Total" data window.

5. To reset the Measurement tool and clear the data values in the measurement data window click on the Clear Select button on the toolbar.

You can successively select points on the map and the tool will record the total distance between the points. This capability might be used to measure the perimeter of an area of interest or the total length of a segmented feature like a transmission line. Figure 23 illustrates the use of the tool to measure the transmission distance from a water energy resource site to the town of Pinon, AZ over an existing transmission line. (Last 3.86 mile segment from the transmission line to Pinon is not highlighted to show segment readout.)

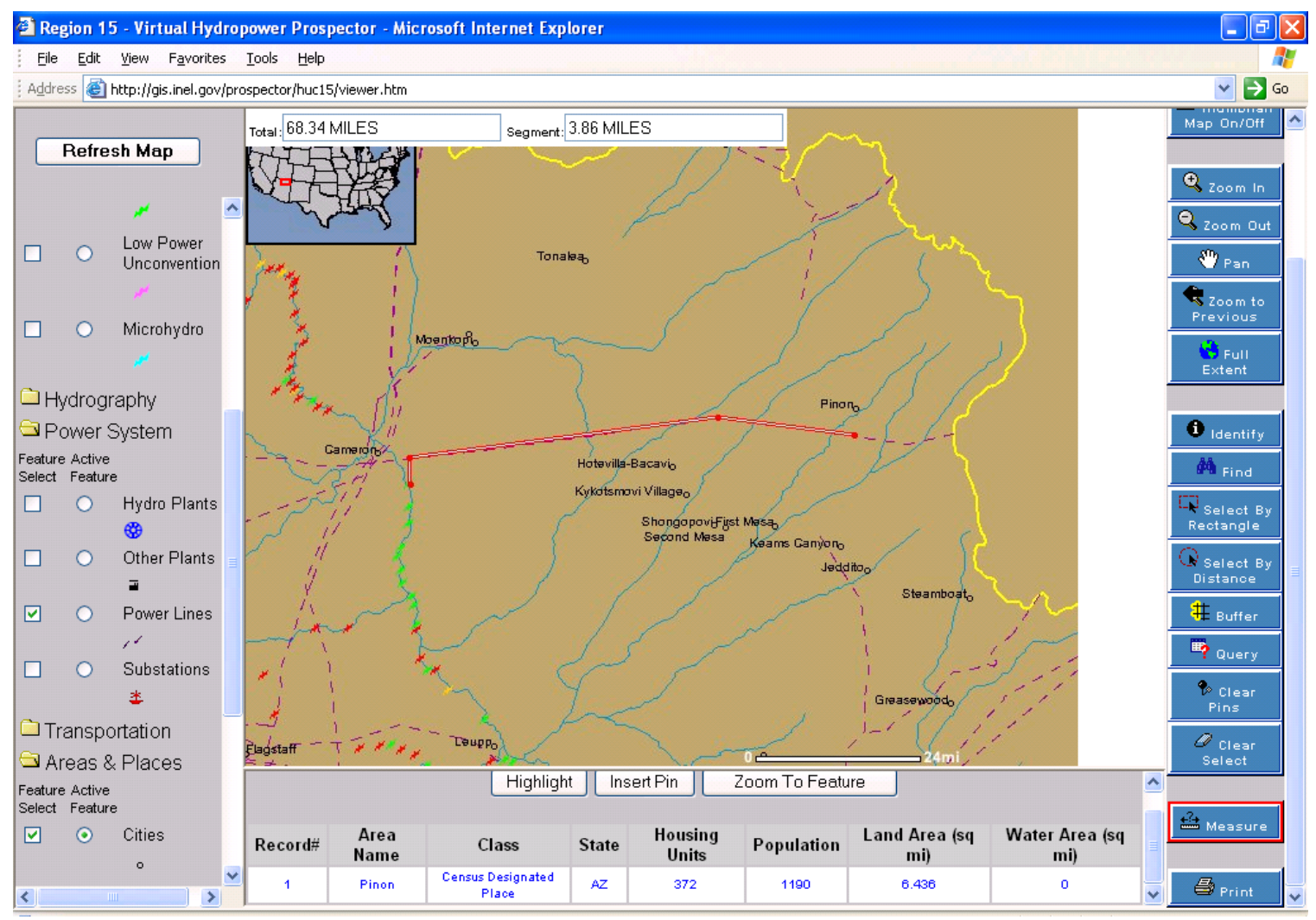

Figure 23. Measurement tool map view display and data readout window.

\subsubsection{Printing a Map}

\section{Print}

The Print tool is used to create a printable image of the map view that is being displayed at the time you click the Print button. To use the tool, do the following:

1. Click on the Print button. The map image specification entry window shown in Figure 24 will appear in the Information Window below the map view. To make a map image specification using the map image specification entry window, do the following: 
a. Click on the "Print Thumbnail Map:" checkbox, if you want a thumbnail map to appear in the printable image.

b. Click on the "Print Legend:" checkbox, if you want a legend to appear in the printable image.

Note: The legend that appears in the printable image will contain only the names and symbols for feature sets that are actually being displayed in the map view when you click on the Print button. The legend in the printable image is a continuous list of features without the folders in the VHP legend.

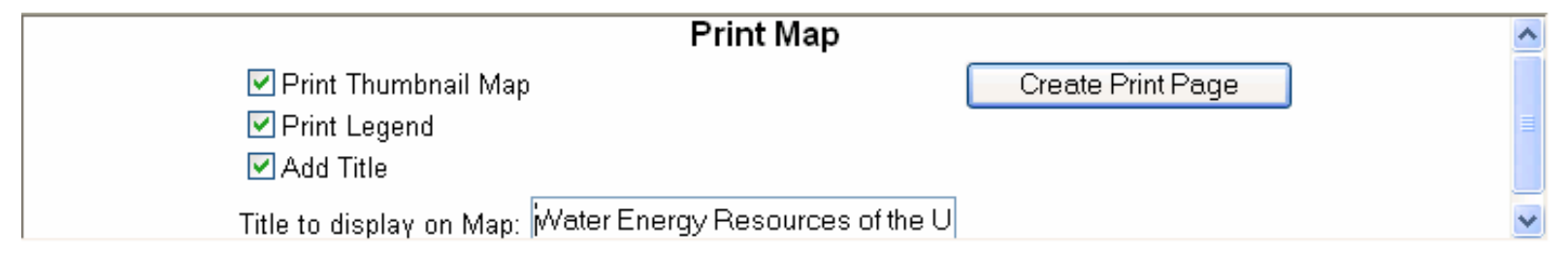

Figure 24. Map image specification entry window.

c. Click on the "Add Title:" checkbox, if you want to title to appear in the printable image. Clicking on the "Add Title:" checkbox opens a window in which you can enter the map title.

2. When you have completed entry of the map image specifications, click on the Create Print Page button. This will open a new window containing the printable map image.

3. Select File in your browser toolbar and then Print in the drop down menu.

4. In the printer settings window, select Properties and then select Landscape as the page setup.

5. Click on the $\mathbf{O K}$ button in the printer settings window to send the print image to the printer.

Note: The image size and resolution of 600 pixels wide by 450 pixels high produced by this tool are fixed. For most map views, the map image and associated legend will fit on $81 / 2$ in. $x 11$ in. paper. The image size is not automatically enlarged when a larger paper size is chosen. For larger than $81 / 2 \mathrm{in} . x 11 \mathrm{in}$. paper sizes, the image size must be increased to fit the paper. Because the resolution of the image is fixed at $600 \times 450$ pixels, image graininess will become noticeable when large paper sizes are used. See Section 3.5.6 below for instructions on printing on larger paper sizes.

A sample map produced using the Print tool is shown in Figure 25. Each of the elements of the composite graphic: map, title, legend, and thumbnail map are separate images and can be resized, relocated, or deleted. 


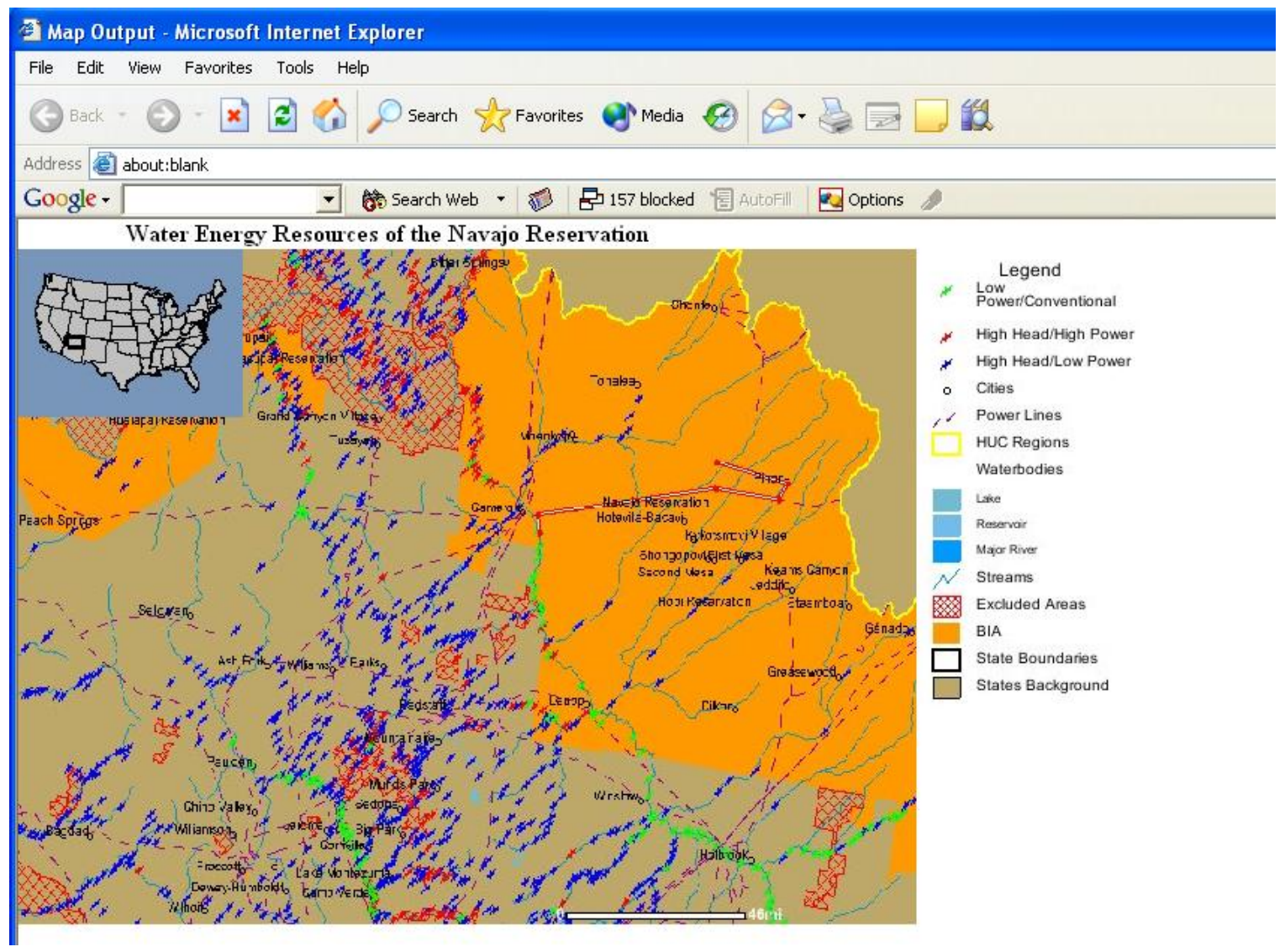

Figure 25. Sample map with title, legend, and thumbnail map produced by the Print tool.

\subsubsection{Including a Map Image in a Document or Slide}

To insert the map image into a document or slide, do the following:

1. Create the map image as described in Section 3.5.5.

2. Be sure that the map image window that is opened when you click on the Create Print Page button is the active window.

3. Right click on the map image and select Copy.

Note: Instead of clicking on Copy you can click on Save Picture As. Selecting this option will allow you to save the map image as a jpeg file in the directory of your choice.

4. Go to the document or slide and click Paste. The map image in the document or slide can then be sized by right clicking on the image, clicking on Format Picture, and choosing the Size tab. When sizing the image, be sure the Lock Aspect Ratio box is checked or the map image will be distorted.

Note: The map image can be printed on larger paper than $81 / 2$ in. $x 11$ in. by pasting the image into a PowerPoint slide with the slide size set to the desired paper size using the Page Setup window accessible by clicking on File in your browser toolbar. The map image can also be printed on larger size paper by opening the map image saved as a jpeg file in a suitable application such as Windows Picture and Fax Viewer. 


\section{CONTACTS}

For further information or comments, please contact:

Sera White

VHP Developer

Idaho National Laboratory

P.O. Box 1625, MS 2213

Idaho Falls, ID 83415-2213

Phone: (208)526-1632

E-mail: sera.white@inl.gov

Douglas G. Hall

Project Manager

Small Hydropower Resource Assessment and Technology Development Project

Idaho National Laboratory

P.O. Box 1625, MS 3850

Idaho Falls, ID 83415-3850

Phone: (208)526-0925

E-mail: douglas.hall@inl.gov 